\title{
Spatial distribution of carbon dust in the early solar nebula and the carbon content of planetesimals
}

\author{
Hans-Peter Gail ${ }^{1}$ and Mario Trieloff ${ }^{2,3}$ \\ 1 Institut für Theoretische Astrophysik, Zentrum für Astronomie, Universität Heidelberg, Albert-Ueberle-Str. 2, 69120 Heidelberg, \\ Germany \\ e-mail: gail@uni-heidelberg.de \\ 2 Institut für Geowissenschaften, Universität Heidelberg, Im Neuenheimer Feld 236, 69120 Heidelberg, Germany \\ ${ }^{3}$ Klaus-Tschira-Labor für Kosmochemie, Universität Heidelberg, Im Neuenheimer Feld 236, 69120 Heidelberg, Germany
}

Received 21 January 2017 / Accepted 22 July 2017

\begin{abstract}
Context. A high fraction of carbon bound in solid carbonaceous material is observed to exist in bodies formed in the cold outskirts of the solar nebula, while bodies in the region of terrestrial planets contain only very small mass fractions of carbon. Most of the solid carbon component is lost and converted into CO during the spiral-in of matter as the Sun accretes matter from the solar nebula.

Aims. We study the fate of the carbonaceous material that entered the proto-solar disc by comparing the initial carbon abundance in primitive solar system material and the abundance of residual carbon in planetesimals and planets in the asteroid belt and the terrestrial planet region.

Methods. We constructed a model for the composition of the pristine carbonaceous material from observational data on the composition of the dust component in comets and of interplanetary dust particles and from published data on pyrolysis experiments. This material entered the inner parts of the solar nebula during the course of the build-up of the proto-sun by accreting matter from the proto-stellar disc. Based on a one-zone evolution model of the solar nebula, we studied the pyrolysis of the refractory and volatile organic component and the concomitant release of hydrocarbons of high molecular weight under quiescent conditions of disc evolution, while matter migrates into the central parts of the solar nebula. We also studied the decomposition and oxidation of the carbonaceous material during violent flash heating events, which are thought to be responsible for the formation of chondrules. To do this, we calculated pyrolysis and oxidation of the carbonaceous material in temperature spikes that were modeled according to cosmochemical models for the temperature history of chondrules.

Results. We find that the complex hydrocarbon components of the carbonaceous material are removed from the disc matter in the temperature range between 250 and $400 \mathrm{~K}$, but the amorphous carbon component survives to temperatures of $1200 \mathrm{~K}$. Without efficient carbon destruction during flash-heating associated with chondrule formation, the carbon abundance of terrestrial planets, except for Mercury, would be of several percent and not as low as it is found in cosmochemical studies. Chondrule formation seems to be a crucial process for the carbon-poor composition of the material of terrestrial planets.
\end{abstract}

Key words. protoplanetary disks - astrochemistry - meteorites, meteors, meteoroids - planets and satellites: composition solid state: volatile

\section{Introduction}

Models of the composition of interstellar dust require that a fraction of about $50 \%$ of the carbon content of the interstellar matter is bound in a solid phase (e.g. Mathis et al. 1977; Zubko et al. 2004). This phase is most likely an amorphous form of solid carbon. Additionally, up to $10 \%$ of the carbon is bound in large polycyclic aromatic hydrocarbons (PAHs). A much more detailed model for the volatile carbonaceous component of the interstellar medium (ISM) dust is developed in Jones et al. (2013, 2014), Jones (2016), Köhler et al. (2015), and Ysard et al. (2015). An overview on this model is given in Jones et al. (2017). The solid material of accretion disks around newly formed stars is derived from such material, although this material is probably somewhat modified in the parent molecular cloud and during the collapse phase to the proto-star before it is added to the disc. The dust component of an accretion disc is therefore expected to contain a considerable fraction of carbonaceous material. By carbonaceous material we mean any solid material that chemically is composed mainly of $\mathrm{C}$ alone or in combination with the elements $\mathrm{H}, \mathrm{N}, \mathrm{O}, \mathrm{S}$, but not compounds with typical rock-forming metals.

It is therefore expected that in particular the material from the cool outer regions of the solar nebula that has not yet been subject to significant chemical and physical processing in the warm inner disc regions is rich in solid carbonaceous materials. This expectation is corroborated by studies of infrared spectra of cometary comae, by direct studies of cometary material of comet Halley and comet Wild 2, and by studies of interplanetary dust particles (IDPs) and micrometeorites. These studies all find high abundances of carbonaceous material (see the reviews of Wooden 2002, 2008; Ehrenfreund et al. 2004). Moreover, carbonaceous chondrites, which are the most pristine meteoritic material, contain some mass-percent of carbonaceous material (Grady \& Wright 2003).

In contrast to this, bodies of the inner solar system ( $₫ 3 \mathrm{AU})$, which is the distance range where terrestrial planets and the parent bodies of the ordinary chondrites are found, are almost void of carbon. The initially abundant condensed carbonaceous material in some way disappeared from this region. Either the carbon 
was converted into gaseous species that are accreted by the Sun and not incorporated into the forming bodies in this region (or only in tiny quantities), or it was first incorporated into the planetesimals, but then converted into volatile species that escape from the bodies and finally are also incorporated in the Sun. In differentiated bodies, it might have been incorporated into the iron core.

The question of how the solid phases of carbon nearly completely disappeared from the disc material in the source region of the terrestrial planets seems not to have attracted much interest. Because in the strongly hydrogen-dominated elemental composition of the solar nebula in chemical equilibrium almost all carbon would be present as gaseous $\mathrm{CO}$ or $\mathrm{CH}_{4}$, it seems at first glance natural that essentially no carbon is built into the terrestrial planets because they form from the solids in the accretion disc. Studies of the chemistry of the accretion disc show unequivocally, however, that the chemistry in the solar nebula and in particular the carbon chemistry is far from chemical equilibrium (e.g. Henning \& Semenov 2013; Walsh et al. 2014). The combustion of carbonaceous material also requires high temperatures because of the high activation energies of key reaction steps, as is known from flame chemistry (see Warnatz et al. 2006). The carbonaceous material that is incorporated into the solar nebula during its formation is therefore not easily converted into gaseous products, which do not contribute to the planetary material in the range of terrestrial planets.

The only work so far that has addressed the question of how the solid carbon phases present in the cool outer regions of the solar nebula could have be converted into gaseous species that are not accreted during planetesimal- and planet-forming processes seems to be the work of Lee et al. (2010). This work discusses photo-processes in the disc atmosphere that ultimately result in oxidation of carbonaceous materials.

We intend to explore in this paper the possibility that the carbonaceous material is converted in the bulk disc close to its midplane by pyrolysis and by chemical reactions. Because a much higher fraction of the disc mass is involved in reactions operating in this region of the disc, these reactions may be more efficient in destroying the carbonaceous material.

The plan of our paper is as follows: in Sect. 2 we discuss the empirical data gained from comets and IDPs on the composition and morphology of solid carbonaceous material in the cold outer parts of the solar nebula. In Sect. 3 we give a sketch of the processes that may be responsible for destruction of the carbonaceous material. In Sect. 4 we outline a simplified model composition of the volatile and refractory components of the carbonaceous material and discuss in Sect. 5 the pyrolysis and oxidation of this material and the subsequent gas-phase reactions. This chemical model is applied in Sect. 6 to a quiescent solar nebula with mass accretion. In Sect. 7 we consider the role that chondrule formation plays for the fate of the carbonaceous material in the solar nebula. In Sect. 8 we discuss the consequences of our results for the carbon content of the parent bodies of meteorites and for planets. Section 9 contains some final remarks. In three appendices we briefly discuss the pyrolysis reactions of the carbonaceous material, the gas-phase reactions following the destruction of carbonaceous material, and present results of a sample calculation.

\section{Carbonaceous material in the solar nebula}

The most direct information we currently have on the pristine composition has been collected from comets. This clearly is unmodified material from the accretion disc (Mumma \& Charnley 2011). Another source of information is the material found in anhydrous chondritic IDPs. This material is generally assumed to be of cometary origin and is likely to have been only slightly modified during its passage through the atmosphere.

Chondrites also contain sometimes a very pristine material that might be traced back to the parent molecular cloud (e.g. Alexander et al. 2007), but generally, the material of chondrites has been chemically processed by parent-body processes, and information from this source is only partly suitable for our purpose. The very detailed model on the structure of the interstellar carbonaceous dust component developed during the past years (see Jones 2016, for a detailed review) is also only of limited use for our purpose. This material is modified in the hot molecular core during the collapse of a molecular cloud core and during passage through the accretion shock until it is added to the accretion disc. Subsequently, it is subject to chemical and thermal processing during its residence in the disc until it is built into planetesimals.

\subsection{Analysis of cometary material}

During the apparition of comet Halley in 1986, an armada of spacecraft were sent to a rendezvous with the nucleus of the comet. Three of the spacecraft succeeded in a close flyby at the nucleus and investigated the elemental, isotopic, and chemical composition of dust grains by their on-board dust-impact timeof-flight mass spectrometers (cf. e.g. Jessberger 1999, for a review). Over 5000 dust grains were analysed.

The composition of carbonaceous dust particles from comet Halley is discussed in Fomenkova et al. (1994) and reviewed by Fomenkova (1997, 1999). According to these studies, the carbon found in solid materials (dust particles) is distributed over a number of phases with different compositions.

1. Most of the carbonaceous material, a fraction of $\sim 0.6$ by total number of carbon atoms in solid phases, is found in what has been called complex organic matter. This is a complex refractory material containing $\mathrm{C}, \mathrm{H}, \mathrm{N}, \mathrm{O}$, and $\mathrm{S}$ with an average elemental composition $\mathrm{C}_{100} \mathrm{H}_{115} \mathrm{~N}_{9} \mathrm{O}_{65} \mathrm{~S}$ (Fomenkova \& Chang 1994). The oxygen in this material may be found in hydroxyl, carbonyl, ether, or carboxyl groups. The nitrogen may be found in amine or nitrile groups. The exact composition of this mixture of a large number of compounds, however, cannot be determined from the type of data that were acquired, but the structure of the insoluble organic matter in meteorites (see Derenne \& Robert 2010 , for an attempt to characterise this) may give an impression of how the chemical structure of the material could look like. It may be that this material resembles the hydrocarbons of the next group with the oxygen- and nitrogen-bearing groups added to them.

2. The second most abundant component is a phase composed of hydrocarbons, containing a fraction of $\sim 0.1$ of all carbon in solids (Fomenkova et al. 1994). It contains compounds of $\mathrm{C}$ and $\mathrm{H}$ and is composed of (1) a component formed by hydrogenated amorphous carbon or polycyclic hydrocarbons with an average $\mathrm{H} / \mathrm{C}$ ratio of 0.14 ; (2) a mixture of PAHs with an average $\mathrm{H} / \mathrm{C}$ ratio of 0.5 ; (3) a component formed by a mixture of PAHs and highly branched aliphatic hydrocarbons with an average $\mathrm{H} / \mathrm{C}$ ratio of 1.0 ; and (4) a component formed from highly branched aliphatic hydrocarbons with a typical $\mathrm{H} / \mathrm{C}$ ratio of about 2.0 . 
3. An almost pure carbon component contains a fraction of $\sim 0.10$ of carbon in solids (Fomenkova et al. 1994). Other elements are contained at a level of at most $1 \%$. Some of such particles have high ${ }^{12} \mathrm{C} /{ }^{13} \mathrm{C}$ isotopic ratios, which means that the presolar carbon grains are members of this phase. The carbon particles are generally very small $(<0.05 \mu \mathrm{m}$, see Fomenkova 1999).

4. The remaining fraction of the carbon was found to be bound in some kind of carbon suboxide ( 0.01, Fomenkova et al. $1994)$ and cyanopolyynes ( 0.01, Fomenkova et al. 1994). We do not consider these low-abundance components.

These carbonaceous components may, at least in part, be inherited from the ISM and/or the parent molecular cloud. The expected structure and composition of the carbonaceous component of the interstellar dust is discussed in detail in Jones et al. (2013, 2014), Jones (2016), Köhler et al. (2015), and Ysard et al. (2015), who built a model based on reasonable dust analogues and on laboratory work on their properties and on their processing under interstellar conditions. The expected similarity, in particular of the complex organic matter of comet Halley, the insoluble organic matter in carbonaceous chondrites, and interstellar carbonaceous dust suggest that they are closely related materials. Possibly part or even all of the complex organic matter of comet Halley and of the insoluble organic matter in carbonaceous chondrites is material from the parent molecular cloud (see e.g. Alexander et al. 2007; Alexander 2011, for a discussion). It would be interesting and useful to study the compositional relation between the carbonaceous components in pristine ISM dust and the dust from the solar nebula, but this is not the topic of this paper. Nevertheless, a rough constraint on the fraction of surviving interstellar grains can be obtained from the abundance of pre-solar or circumstellar grains found in primitive meteorites or interplanetary dust particles. In primitive meteorites and also in IDPs, which belong to the most primitive matter considered in our paper, certain refractory pre-solar grains have quite low abundances. $\mathrm{SiC}$, for instance, ranges from a few to circa $100 \mathrm{ppm}$, graphite to circa 5-10 ppm, and only non-carbonaceous silicates have average abundances of $400 \mathrm{ppm}$ in IDPs, with exceptional cases reaching a few $1000 \mathrm{ppm}$. This means that even the fraction of surviving refractory pre-solar material seems at best of the order of a few permil (see e.g. McSween \& Huss 2010). As these grains are more resistant against chemical destruction than more volatile components, a few permil seems to be an upper limit for the fraction of surviving volatile components. Moreover, the mere presence of bulk isotopic anomalies in $\mathrm{D} / \mathrm{H}$ does not imply that the carrier phases were not chemically modified. It just means that their isotopic ratio did not equilibrate completely with other material that had the bulk solar system value. Hence, we share the view of many meteoriticists that most of the solar system material was homogenised in the proto-solar disc or at least chemically modified.

The abundance of carbon relative to $\mathrm{Si}$ and its fraction bound in carbonaceous solid material was studied by Geiss (1987). According to this study, the $\mathrm{C} / \mathrm{Si}$ abundance ratio is close to solar. The solar abundance of carbon had to be revised downwards since then (Asplund et al. 2009), but in view of the considerable uncertainties of abundance determinations for comet Halley, the basic statements remain unchanged. In the material released by comet Halley, a fraction of $\sim 0.47$ of the carbon is bound in the solid carbonaceous phases while the remaining fraction is found in the released gas (Geiss 1987). It has to be noted that the dust grains from comet Halley were analysed a few hours after their release when they were already heated to a high temperature by illumination from sunlight for some hours, such that the more volatile condensed phases evaporated. That some of the less-refractory carbonaceous material is desorbed before measurement by the space probes is suggested by the results for the distribution of gas species in the coma of comet Halley, which seems to require that the coma has an extended source of carbon (Eberhardt et al. 1987; Cottin \& Fray 2008).

Attempts have been undertaken to identify PAHs in the coma spectrum of comet Halley, and it has been claimed that pyrene $\left(\mathrm{C}_{16} \mathrm{H}_{10}\right)$ and anthracene $\left(\mathrm{C}_{14} \mathrm{H}_{10}\right)$ are detected by emission bands in the UV (Clairemidi et al. 2004, 2008). The results are inconclusive, however. Such PAHs were successfully identified in carbonaceous material from dust particles collected in the tail of comet Wild 2 (Clemett et al. 2010); compare also the review of Li (2009). This shows that PAHs are indeed present in cometary material.

The carbonaceous material in most cases seems to form a coating on a chondritic core (Kissel \& Krueger 1987).

The results derived from observations of comet Halley are much more conclusive than inferences from of the carbon content of particles of comet Wild 2 (Brownlee et al. 2006) because these particles were captured at high speed in an aerogel and may have experienced significant loss of volatiles, including carbon, during the collection process.

\subsection{Laboratory analysis of interplanetary dust particles}

Interplanetary dust particles are typically $10 \mu \mathrm{m}$ sized aggregates of a large number of mineral grains $\left(>10^{4}\right)$ and carbonaceous material. They show fluffy structures of very small grains with diameters of between 1 and $150 \mathrm{~nm}$. They are composed of glass, crystalline mineral grains, and Fe, Ni-metal and Fe-rich sulphide crystals embedded in glass, with abundant carbonaceous material apparently acting as a glue. The porosity of these aggregates is very high, between 0.7 and 0.94 (Flynn et al. 2013).

The IDPs are enriched by carbon and other moderately volatile elements by factors of around two to three compared to CI chondrites (Flynn et al. 1996). Some IDPs show strongly anomalous $\mathrm{D} / \mathrm{H}$ isotopic ratios, suggesting that they incorporated molecular cloud material that pre-dates the solar nebula (e.g. Keller et al. 2000). After their formation, they were obvious never heated to temperatures sufficient for evaporation of the moderately volatile elements (Flynn et al. 1996). The subclass of anhydrous chondritic porous interplanetary dust particles (CPIDP) therefore apparently represents the most primitive available material from the early days of solar system formation (cf. the review of Messenger et al. 2006). They are most likely derived from comets from the Kuiper-belt region, such that their composition can probably be taken as being close to the initial composition of solar nebula solids. The presence of some fraction of crystalline material (Bradley 1994) means, however, that they also contain some fraction of material from the inner parts of the solar nebula, such that their composition is not completely identical to the composition of the material that rained down from the parent molecular cloud of the solar system.

The carbon content of the IDPs is highly variable and amounts on average to 12 weight-percent (Thomas et al. 1994) and may be as high as $46 \%$ (Thomas et al. 1993). The volume fraction of the carbonaceous material is between a few percent and up to $90 \%$ (Flynn \& Keller 2003). The spatial distribution of the carbonaceous material within IDPs was investigated by scanning transmission X-ray microscope (STXM; cf. 
Flynn et al. 2003, 2004). The carbon is observed to form three morphologically different structures in IDPs.

- Thin coatings $(\sim 100 \mathrm{~nm})$ on individual grains. The individual grains are generally not in contact with each other, but separated by such a coating. A very instructive picture is shown in Flynn et al. (2013, Fig. 2).

- Discrete sub- $\mu \mathrm{m}$ to $\mu \mathrm{m}$ carbonaceous regions.

- Thick $(\sim 0.5 \mu \mathrm{m})$ coatings on aggregates of grains, acting as a "glue" that appears to hold the subunits together.

According to Flynn et al. (2003), the fact that carbon occurs as coatings on grains as the material that holds the subunits of the IDPs together, indicates that this carbon existed already at the time when grains were being assembled into dust-sized particles. The morphology of the carbonaceous material in IDPs therefore shows the initial form of the appearance of solid carbon in the solar nebula.

The composition of the carbonaceous material was investigated by X-ray Absorption Near Edge Structure (XANES) spectroscopy and by Fourier transform infrared spectroscopy (FTIR; e.g. Flynn et al. 2003, 2004, 2013). The results of interest for us are:

1. From XANES it is found that the abundance of purely amorphous carbon is low. A characteristic feature for amorphous carbon in the K-edge XANES spectrum of $\mathrm{C}$ was found for only 1 of the 12 investigated particles (Flynn et al. 2003).

2. Most carbon is present as some type of organic material, as witnessed by a high oxygen content of the carbonaceous material (Flynn et al. 2003). This was detected by XANES of the $\mathrm{K}$-edge of $\mathrm{C}$, which revealed strong absorption features that were identified with abundant $\mathrm{C}=\mathrm{O}$ bonding and with $\mathrm{C}=\mathrm{C}$ bonding in ring structures of the $\mathrm{C}$ atoms (Flynn et al. 2003 , 2013). The absence of a feature characteristic of graphitic carbon indicates that this form of condensed carbon is (nearly) absent (Flynn et al. 2004).

3. By FTIR spectroscopy of the $3 \mu \mathrm{m}$ regime, it was found that of the 19 IDPs investigated, 17 showed strong C-H stretching vibrations of aliphatic hydrocarbons, which are associated with $\mathrm{CH}_{2}$ and $\mathrm{CH}_{3}$ groups (Flynn et al. 2003). The relative strength of the features is highly variable for different particles, indicating a variable mixture of different aliphatic hydrocarbons. The average value of the $\mathrm{CH}_{2} / \mathrm{CH}_{3}$ ratio is $\sim 2.46$ for anhydrous IDPs (Flynn et al. 2003). Since for simple aliphatic hydrocarbons the $\mathrm{CH}_{3}$ groups terminate the chains of $\mathrm{CH}_{2}$ groups, the $\mathrm{CH}_{3} / \mathrm{CH}_{2}$ ratio is a measure of the chain length of the aliphatic hydrocarbons. This depends strongly on the unknown branching ratio and is not easily converted into a chain length, however.

The mass fraction of aliphatic hydrocarbons in IDPs was estimated in Flynn et al. (2004) to be approximately 1 to 3 weightpercent. Since the total carbon abundance is on average 12 weight-percent, the aliphatic hydrocarbons comprise a fraction of $\sim 0.1 \ldots 0.2$ of the carbon.

4. It is reported that only a few of the investigated IDPs showed C-H stretching vibrations characteristic of aromatically bound $\mathrm{C}$, and these are found to be weak. The fraction of aromatically carbon in IDPs therefore seems to be low (Flynn et al. 2003).

5. For one IDP, Flynn et al. (2008) succeeded to determine the composition of the organic coating of one of the silicate grains. Using XANES, the authors derived an average composition of $\mathrm{C}_{100} \mathrm{~N}_{10} \mathrm{O}_{50}$.
These findings with respect to the composition of the carbonaceous material in IDPs are essentially in accord with the results found from analysis of dust of comet Halley. Since the anhydrous IDPs originate almost certainly from a number of different comets with different formation regions in the solar nebula, we have to conclude that the refractory condensed phases of carbon have a rather uniform composition in the outer parts of the solar nebula, despite the significant particle-to-particle variations. Except for the small fraction of pure carbon grains, most of the refractory carbon phases are found in aggregate dust particles as coatings on mineral dust grains.

\section{Metamorphosis of the carbonaceous material in the solar nebula}

Our main interest is the carbon content of the central parts of the solar nebula. The terrestrial planets and parent bodies of the ordinary chondrites are formed in this region. During the early evolutionary phase of an accretion disc like the solar nebula, a general net flow of matter through the inner disc toward the proto-sun exists; this is called the accretion flow. The material that is accreted by the still-growing sun from the inner edge of the disc is continuously replenished by material that is initially located farther out in the disc. The residence time of the material in the inner disc is estimated to be shorter than $10^{5} \mathrm{yr}$, assuming that the mass contained in the inner disc region is of the order of $10^{-3} M_{\odot}$ and the accretion rate is a few times $10^{-8} M_{\odot} \mathrm{yr}^{-1}$ (which may be typical values during the initial evolution period, Manara et al. 2012; Da Rio et al. 2014). Planetesimal formation commences at probably a few times $10^{5} \mathrm{yr}$ after disc formation in the innermost part of the solar nebula and at about $1 \ldots 2 \times 10^{6} \mathrm{yr}$ at the distances of the present asteroid belt. At this instant, the material in the inner disc region is no longer identical with the material that was deposited in this region during the initial formation period of the accretion disc. Instead, it is material that originates from cold disc regions and migrated inwards during the course of the accretion process.

In the cold outer regions of the accretion disc, where water ice is frozen, the composition of the refractory solids is maintained in the state it has been since the time when the matter rained down to the accretion disc from the parent molecular cloud of the Sun. Temperatures are too low in this region for any physical or chemical modification of the minerals. It is not clear whether this also holds for the refractory carbonaceous components. The type of material found in IDPs shows a composition that seems to be different from what is assumed for the composition of the interstellar carbon dust. The corresponding compositional changes ought to occur either in the parent molecular cloud or in the accretion disc itself. The high $\mathrm{D} / \mathrm{H}$ isotopic ratios found in the carbonaceous material of some IDPs are interpreted as pointing to an origin of at least part of the carbonaceous material in the extremely cold environment of a giant molecular cloud (e.g. Alexander 2011), but if such material can be formed in the parent molecular cloud, it can very likely also be formed in the cold outer parts of the accretion disc, where similar conditions are encountered with respect to the chemistry acting as in molecular clouds. It has been argued (Derenne \& Robert 2010) that the insoluble organic matter in carbonaceous chondrites, which must somehow be derived from the carbonaceous matter in IDPs, is of solar nebular origin, and not from the ISM.

For our purposes, the question of the true origin of the carbonaceous material is not important. We only need to know the composition and morphology of the carbonaceous material as it 


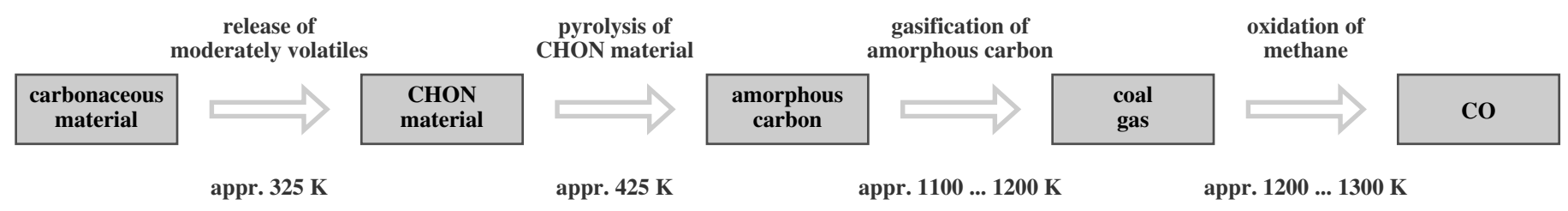

Fig. 1. Processes responsible for the conversion of the carbonaceous material observed for cool disc regions into gaseous components, and their final oxidation to $\mathrm{CO}$.

migrates into the inner disc region. At this place, we take recourse to what is learned from investigations of cometary particles and IDPs. These showed that the carbonaceous component of the dust aggregates is composed of four essentially different components:

1. Pure amorphous carbon grains.

2. An aliphatic hydrocarbon component.

3. An aromatically bound hydrocarbon component.

4. Refractory carbonaceous material with rather high contents of $\mathrm{O}, \mathrm{N}$, and $\mathrm{S}$ and with a poorly defined composition.

The second, third, and fourth component form a coating on the mineral grains. This mixture of carbon compounds defines the initial composition of the fraction of the carbon that enters the inner part of the solar nebula in the form of refractory condensates. The remaining fraction of carbon is in volatile ices and in the gas phase (CO). More details are specified below.

During the evolution of the accretion disc, there is a general average slow inflow of matter from cold outer regions towards the centre. The general temperature structure of the disc during the about first million years of its evolution is such that the temperature in the interior of the disc increases with decreasing distance from the proto-sun. If we refrain for the moment from the more complex local flows superposed on this long-term accretion flow, the matter is systematically transported by inflow into regions of increasingly higher temperature, where chemical reactions are activated and finally reaction timescales become short enough to establish chemical equilibrium.

As long as the dust component of the disc matter is not separated from the gas and enclosed in planetesimals, it is chemically coupled to the gas phase. The highly fluffy structure observed for IDPs means that the elementary building blocks of the dust aggregates are all in direct contact with the gas phase. The chemical compositions of the dust and gas components evolve simultaneously during gradual heating caused by inward migration of the matter. They are coupled by solid-gas interface reactions. If planetesimals are formed from the solid component of disc matter in some region of the accretion disc, their initial composition corresponds to the local composition of the solids at this instant and in this region. The subsequent evolution of the internal chemical composition of planetesimals and of the residual gaseous material in the disc follow different paths from this moment on.

Our interest is on the question of how much of the initial refractory carbon dust material still exists in different zones of the solar nebula that can be incorporated into planetesimals and later into planetary bodies. To answer this, we have to study the evolution of the initial material as it is heated during inwards migration. The individual carbon compounds constituting the initial material differ in their volatility. The aliphatic hydrocarbons found in cometary material are moderately volatile. They are transferred to the gas phase at rather low temperature by evaporation. The small fraction of amorphous pure carbon is highly refractory. This component survives up to rather high temperatures (>1000 K, see Gail 2001; Wehrstedt \& Gail 2002) until it is destroyed by oxidation reactions and is finally converted into $\mathrm{CO}$. The main part of the carbonaceous material is a refractory organic material probably similar to the material kerogen known from Earth, which evaporates and decomposes at several hundred $\mathrm{K}$, forming gaseous hydrocarbons and a residue resembling amorphous carbon.

The conversion of the refractory carbon into gaseous species enriches the gas phase with hydrocarbons. In chemical equilibrium, these would be converted into $\mathrm{CO}$ and $\mathrm{CO}_{2}$. Since the gasphase reactions for oxidation of the hydrocarbons involve relatively high activation energy barriers, the oxidation reactions require rather high temperatures to become efficient $(\gtrsim 1200 \mathrm{~K}$ in the solar nebula, Gail 2002). These barriers appear in the dissociation of $\mathrm{H}_{2} \mathrm{O}$ to form reactive $\mathrm{OH}$ radicals in case of accretion discs, or for the dissocation of $\mathrm{O}_{2}$ to form $\mathrm{O}$ radicals in case of terrestrial flames. The $\mathrm{O}$ and $\mathrm{OH}$ radicals then attack hydrocarbons, which leads to the formation of a C-O-bond, and through a chain of follow-up reactions, it ultimately leads to $\mathrm{CO}$ molecules. From laboratory studies on combustion of gaseous fuels, it is known that during the course of such processes, complex organic compounds are to a large extent first degraded into mainly $\mathrm{C}_{2} \mathrm{H}_{2}$ and $\mathrm{CH}_{4}$, and the final oxidation then heavily relies on the hot gas-phase chemistry of such molecules (e.g. Warnatz et al. 2006; Marinov et al. 1996). Hence, there will be a certain zone in the accretion disc where the complex aliphatic and aromatic compounds are converted into simple hydrocarbons of low molecular weight, which finally are converted into $\mathrm{CO}$ in a hotter region.

Hence, the main processes to be considered to study the metamorphosis of carbon in the solar nebula are (cf. Fig. 1)

- evaporation and condensation of the volatile components of the carbonaceous mixture;

- pyrolysis of the refractory organic components;

- gasification of the residual coke by reaction with hot steam (so-called town-gas reaction); and

- final conversion of the hydrocarbons by additional reaction with hot steam to $\mathrm{CO}$.

The combination of these physico-chemical processes with the transport of material in the accretion disc and the gradual evolution of the disc properties determines the fraction of the total carbon that exists as condensed carbonaceous material at any instant and location in the solar nebula, and in turn, this determines the amount of carbonaceous material that is incorporated into planetesimals.

\section{Dust model}

In order to determine the quantities of the different gaseous and condensed carbon-bearing species in the solar nebula, we have to model the chemical reaction processes between all carbon compounds in the gas phase, the exchange of material between the gaseous and condensed material, and the chemical surface reactions. Because of the complex structure and composition of the 
dust, this requires some simplifications. In the following we introduce such a model for the carbonaceous material. The model is guided by what is known about IDPs and cometary dust, and it concentrates on the basic aspects that are likely to be important for modelling the distribution of carbon dust in the warm inner disc regions.

\subsection{Structure of dust particles}

The observed IDPs are complex aggregates of thousands of tiny mineral dust grains with sizes $\lesssim 0.1 \mu \mathrm{m}$, each of which is coated with carbonaceous material, which seems to act as a glue for the aggregate (see Sect. 2). The dust material entering the outer edge of the computational domain is assumed to have the same morphology as is observed for IDPs.

The porosity of the aggregates is very high, with observed values in the range $0.7 \ldots 0.94$ (Flynn et al. 2013). Since the aggregates were slightly under pressure by self-gravity of the cometary parent body and because they are therefore slightly compacted during their residence in the comet (e.g. Henke et al. 2012, Fig. 2), it is likely that the highest porosities found for IDPs resemble their typical porosity before they were initially incorporated into the parent comet. At such high porosities, the aggregates are mainly highly branched linear chains of particles. With respect to gas-grain collisions, the surface of each grain of such a highly fluffy grain aggregate is essentially in direct contact with the gas phase into which the aggregate is suspended. Inside such a fluffy aggregate, the probability that a gas-phase particle experiences multiple collisions with the constituent grains of the aggregate is negligibly small.

To calculate the evaporation and condensation processes or chemical reactions between gas-phase species and surface atoms, this allows us to introduce the simplification that for the purposes of modelling the chemistry (and only for this purpose), each grain of an aggregate is replaced by a hypothetical single isolated grain that interacts with the gas phase. Each of this particle is assumed to be initially coated with a mantle of carbonaceous material, as seems to be the case for the real IDPs. Any previously existing ice coating has already evaporated at the temperatures of interest ( $>200 \mathrm{~K})$. According to Lawler \& Brownlee (1992), neither pure silicate nor pure CHON particles seem to be detected in the dust from comet Halley. For simplicity, we additionally assume that all the carrier grains of the carbonaceous mantles are spheres, and all have the same radius. This radius is arbitrarily set to $50 \mathrm{~nm}$.

In principle, the dust aggregates may drift with respect to the gas. The velocity calculated for the whole aggregates then has to be prescribed for each of its grain members. This becomes important at the stage of evolution where planetesimals are formed. Since the main subject of the present paper is the carbon chemistry in the solar nebula during the period preceding planetesimal formation, however, we here refrain from considering dust drift in our model calculation.

\subsection{Model composition of carbonaceous material}

We assume that the composition of the carbonaceous material found in anhydrous chondritic IDPs and observed for comet Halley is representative of the material that slowly migrates inwards by accretion. The composition of this material is not precisely known, and significant variations are found for different IDPs. Hence, for the purpose of the model computations, a simplified model composition is introduced that represents the essential properties of the material.

Pollack et al. (1994) have previously discussed this problem in the context of calculating opacities for accretion discs. Despite much observational and theoretical efforts to determine the composition of cometary material and great progress in many details (cf. the review of Mumma \& Charnley 2011), the knowledge of the general composition of carbonaceous material has not much improved since the work of Pollack and collaborators. We therefore base our model essentially on the same assumptions as Pollack et al. (1994).

One important parameter is the fraction of the carbon that is bound in the carbonaceous material. This was estimated from the C/Si ratio in particles of comet Halley (Jessberger et al. 1989) by Pollack et al. (1994) to be 0.55 of the total carbon. No better information seems to be available, and this estimate does not significantly change when an improved C abundance (Scott et al. 2015 ) is used (see Bergin et al. 2015). We therefore also assume a fraction of 0.55 of the carbon to be condensed in carbonaceous material. The remaining fraction is in the gas phase, and beyond the ice line, some of this is frozen.

The carbonaceous material is assumed to be composed of the four components discussed in Sect. 2 that carry the fractions of the condensed carbon listed in Table 1 (data taken from Fomenkova 1997). With increasing temperature, these components decompose, evaporate, or are destroyed by oxidation, releasing hydrocarbons to the gas phase. The table also shows the typical release temperatures (discussed below) and our assumptions with respect to the gaseous compounds that are released, which we discuss now.

\subsection{Gasification of carbonaceous material}

An important information for calculating the chemistry is the composition of the gaseous components that are released on heating the material. We describe the corresponding mixture with only a small number of components because the available information is fragmentary.

Amorphous carbon. The oxidation of amorphous carbon is discussed in Finocchi et al. (1997) and Gail (2001). The same reaction model is used here. According to this information, the carbon is oxidised by an attack of $\mathrm{OH}$ radicals (here formed at elevated temperature by thermal dissociation) to carbon atoms at the periphery of aromatically bound carbon and releasing $\mathrm{HCCO}$ molecules to the gas phase. This reaction is irreversible and destroys the carbon.

Aliphatic compounds. The aliphatic and aromatic hydrocarbons found in the carbonaceous material are unlikely to exist there as isolated molecules. For the aliphatic compounds, the average $\mathrm{CH}_{2} / \mathrm{CH}_{3}$ ratio is 2.46 , as determined for IDPs (see Sect. 2.2), which indicates chain lengths for unbranched chains of between 6 and 8 carbon atoms, but the H/C ratio of about 1 found in many cases (see Sect. 2.1) indicates highly branched alkanes. They are likely to be part of more complex networks comprising aliphatically and aromatically bound subunits like the unit reported in Derenne \& Robert (2010).

The outgassing of some laboratory-made analogue materials of this type was studied by Nakano et al. (2003). They found that the more volatile part of this material outgasses at temperatures somewhat above $300 \mathrm{~K}$ and decomposes during 
Table 1. Assumed composition of the carbonaceous material in cold disc regions.

\begin{tabular}{llcccrr}
\hline \hline & & Fraction $f$ & \multicolumn{2}{c}{ Outgassing } & \multicolumn{2}{c}{ Released } \\
Carbonaceous dust component & Structure & of C & Process & Temperature & Species & Percent \\
\hline Pure carbon dust & Amorphous carbon & 0.1 & Oxidation & 1100 & $\mathrm{HCCO}$ & 100 \\
Volatile organic material & Aliphatic compounds & 0.1 & Pyrolysis & 325 & $\mathrm{CH}_{4}$ & 50 \\
& & & & & $\mathrm{C}_{4} \mathrm{H}_{10}$ & 50 \\
Moderately volatile organics & Aromatic compounds & 0.1 & Evaporation & 425 & $\mathrm{C}_{16} \mathrm{H}_{10}$ & 100 \\
Refractory organic material & CHON & 0.6 & Pyrolysis & 425 & $\mathrm{CH}_{4}$ & 15 \\
& & & & & $\mathrm{CO}_{2}$ & 25 \\
& & & & & $\mathrm{CO}_{2}$ & 20 \\
& & 0.1 & & & $\mathrm{C}_{16} \mathrm{H}_{10}$ & 40
\end{tabular}

Notes. The fractions of carbon bound in the different components are given with respect to the total carbon bound in the carbonaceous material, which in turn is a fraction of 0.55 of the $\mathrm{C}$ element abundance. For the different components, we list the gases assumed to be released by heating or oxidation and the corresponding approximate release temperatures (in K) and the relative proportions of the products.

outgassing, releasing short alkanes like $\mathrm{CH}_{4}$ into the gas phase. The release of product gases of low molecular weight that are dominated by $\mathrm{CH}_{4}$ is also observed for heating of terrestrial kerogen (e.g. Wang et al. 2013), although at higher temperatures the outgassing conditions are quite different. Kerogen is often considered as an analogue of the carbonaceous material (cf. Pollack et al. 1994). The somewhat less volatile aromatically bound component of the material investigated by Nakano et al. (2003) outgassed at temperatures of around $450 \mathrm{~K}$, apparently without decomposition.

We assume therefore for our model of the carbonaceous material that the aliphatic component is released to some part (50\%) of the corresponding carbon fraction as $\mathrm{CH}_{4}$ (methane), and that the remaining part is released as $\mathrm{C}_{4} \mathrm{H}_{10}$ (butane) in order to also include a longer alkane chain in our model.

Aromatic compounds. For the aromatic component, the weak evidence from observations (see Sect. 2.2) indicates that released PAHs have up to 16 carbon atoms. We assume in our model for the carbonaceous material that the outgassing of the aromatic component releases $\mathrm{C}_{16} \mathrm{H}_{10}$ (pyrene). This choice is somewhat arbitrary and mainly serves the purpose to include a small $\mathrm{PAH}$ molecule in our model.

Our assumptions with respect to the pyrolysis products of the aromatic and aliphatic components are collected in Table 1.

CHON material. The main components of the carbonaceous material are the $\mathrm{CHON}$ particles with very complex composition. They have rather high contents in $\mathrm{O}, \mathrm{N}$, and $\mathrm{S}$. The average composition of the $\mathrm{CHON}$ particles in comet Halley, for instance, was determined as $\mathrm{C}_{100} \mathrm{H}_{115} \mathrm{~N}_{9} \mathrm{O}_{65} \mathrm{~S}$ (Fomenkova \& Chang 1994), and a composition of $\mathrm{C}_{100} \mathrm{~N}_{10} \mathrm{O}_{50}$ has been reported by Flynn et al. (2008) for the coating of a single grain in an IDP, where the composition of the coating could be determined by lucky circumstances. This means that this component carries a non-negligible fraction of the available oxygen (and also of $\mathrm{N}$ and others, but we do not consider nitrogen). The material of the CHON particle is assumed to be similar to terrestrial kerogen, and we again take recourse to what is observed for kerogen pyrolysis.

Because of the significant oxygen content of the $\mathrm{CHON}$ material, $\mathrm{CO}$ and $\mathrm{CO}_{2}$ are probably important pyrolysis products of the CHON material. The experimental results of
Nakano et al. (2003) for their proxy of the carbonaceous material show that most of the oxygen is released in this way. Some ethanol is also released, but this is neglected in our model to keep the number of components small. The nitrogen content of the carbonaceous material is released in the pyrolysis experiments of Nakano et al. (2003) as $\mathrm{NH}_{3}$. We do not consider any nitrogen and sulphur compounds. We subtract from the average composition of the $\mathrm{CHON}$ material $\left(\mathrm{C}_{100} \mathrm{H}_{115} \mathrm{~N}_{9} \mathrm{O}_{65} \mathrm{~S}\right.$, Fomenkova \& Chang 1994) the $\mathrm{N}$ and $\mathrm{S}$ content and the corresponding number of $\mathrm{H}$ atoms and remain with a $\mathrm{CHO}$ composition of $\mathrm{C}: \mathrm{H}: \mathrm{O}=100: 86: 65$.

The most abundant carbon-bearing highly volatile pyrolysis product of kerogen is $\mathrm{CH}_{4}$, in addition to less abundant other alkanes and alkenes (Wang et al. 2013). Methane is also the most abundant hydrocarbon of low molecular weight in the experiment of Nakano et al. (2003). We assume for simplicity that methane is the sole hydrocarbon pyrolysis product of low molecular weight.

Pyrolysis of terrestrial kerogen results in a significant fraction in the production of oil, that is, in compounds of high molecular weight. For their proxy of the cometary carbonaceous material, Nakano et al. (2003) found that some fraction of the pyrolysis products are hydrocarbons of high molecular weight that did not decompose during evaporation. We assume the component of high molecular weight of the carbonaceous material pyrolysis products to be represented by the same compound (pyrene) as in the case of the aromatic component of the carbonaceous material in order to keep the number of components in our model small.

With the observation that $\mathrm{CO}_{2}$ seems to be somewhat less abundant in the released gases than $\mathrm{CO}$, we find the relative fractions for the pyrolysis products listed in Table 1, which closely reproduce the assumed approximate $\mathrm{C}: \mathrm{H}: \mathrm{O}=100: 86: 65$ ratio for the carbonaceous material.

This is a highly simplified model of the carbonaceous material that attempts to describe some basic features of its decomposition into gaseous products at elevated temperature. The further fate of the product molecules has to be determined by modelling the gas-phase chemistry of hydrocarbons in the solar nebula.

\subsection{Pyrolysis temperature}

The laboratory experiment of Nakano et al. (2003) shows that pyrolysis products are released over some extended temperature 
regime. The light components are released at between about $300 \mathrm{~K}$ and $350 \mathrm{~K}$, and the heavier products disappear from the residue between about $400 \mathrm{~K}$ and $450 \mathrm{~K}$. The data given in the table are confirmed by pyrolysis temperatures derived from kinetic data on the pyrolysis of kerogen, as discussed in detail in Appendix A.1.

This assumption roughly corresponds to the vaporisation temperatures assumed in the dust model of Pollack et al. (1994) for their volatile and refractory organic compounds, although the reasons for the assumed temperatures are somewhat different. Our assumed pyrolysis temperatures are only rather crude estimates. More detailed laboratory investigations are necessary to obtain more precise information on the pyrolysis of the carbonaceous material.

\section{Evolution of the carbonaceous material}

The models of Gail (2001) and Wehrstedt \& Gail (2002) have included the oxidation of amorphous carbon dust. In the present model calculation, the method for modelling soot oxidation is the same as described in the previous papers, and therefore the details are not repeated.

In addition to the amorphous carbon, we have to consider additional carbonaceous components, which show a different thermal evolution. According to our model for the carbonaceous material specified in Table 1, we have three additional components. It is assumed that these additional components form coatings on the surface of the mineral dust grains (see Sect. 4.1), and we assume that these cores all have the same radius $a_{\mathrm{c}}$. If the thickness of the coatings is not substantially larger than the core radius, we may neglect the change in surface area during the gasification of the carbonaceous material and assume some average surface area to calculate reaction rates. Then it suffices to consider only the change of the total quantities of the material and not the details of the evolution of the coating.

Since the gasification of the components releases different gaseous species, it is useful to think of each component as a collection of sub-components with the composition of the corresponding released gas-phase species. For instance, the volatile organic material is assumed to release $\mathrm{CH}_{4}$ and $\mathrm{C}_{4} \mathrm{H}_{10}$ upon pyrolysis. We introduce two species $\mathrm{CH}_{4}[\mathrm{v}]$ and $\mathrm{C}_{4} \mathrm{H}_{10}$ [v] that represent the $\mathrm{C}$ and $\mathrm{H}$ atoms bound in the volatile carbonaceous component that are finally released as $\mathrm{CH}_{4}$ and $\mathrm{C}_{4} \mathrm{H}_{10}$ molecules to the gas phase. Then the pyrolysis process can be described by the chemical reaction equations

$$
\mathrm{CH}_{4}[\mathrm{v}] \longrightarrow \mathrm{CH}_{4}, \quad \mathrm{C}_{4} \mathrm{H}_{10}[\mathrm{v}] \longrightarrow \mathrm{C}_{4} \mathrm{H}_{10}
$$

Analogously, we define components $\mathrm{C}_{16} \mathrm{H}_{10}[\mathrm{~m}]$ for the moderately volatile organics and $\mathrm{CH}_{4}[\mathrm{r}], \mathrm{CO}[\mathrm{r}], \mathrm{CO}_{2}[\mathrm{r}]$, and $\mathrm{C}_{16} \mathrm{H}_{10}[\mathrm{r}]$ for the refractory organic material.

The quantities of each of these components at each location and instant is described by a fictitious concentration $c_{i}^{(\mathrm{s})}$ of this species per $\mathrm{H}$ nucleus. The initial concentrations in the lowtemperature regime of the disc are by definition

$c_{0, i}^{(\mathrm{s})}=\frac{f_{i} \gamma_{i}}{n_{i}} \epsilon_{\mathrm{C}}$

where $\epsilon_{\mathrm{C}}$ is the element abundance of $\mathrm{C}$ with respect to $\mathrm{H}$ in the solar nebula, $f_{i}$ is the fraction of carbon initially bound in the corresponding carbonaceous component to which $i$ belongs, as given in the third column of Table 1 , and $\gamma_{i}$ is the fraction of $\mathrm{C}$ atoms released as the particular molecule considered as given in the last column of Table 1 . Finally, $n_{i}$ is the number of $\mathrm{C}$ atoms in the chemical formula of the species.

The concentrations $c_{i}^{\text {(s) }}$ vary with location within the disc and with time by transport and mixing by large-scale flows and turbulent diffusion, and they vary by pyrolysis of the carbonaceous material at elevated temperatures. We therefore add to the set of diffusion-transport-reaction equations described in Gail (2001), Wehrstedt \& Gail (2002) additional equations for the new components. In cylindrical coordinates and averaged over the vertical direction, the equations take the form

$$
\frac{\mathrm{d} c_{i}^{(\mathrm{s})}}{\mathrm{d} t}=\frac{\partial c_{i}^{(\mathrm{s})}}{\partial t}+v_{r} \frac{\partial c_{i}^{(\mathrm{s})}}{\partial r}=\frac{1}{h n_{\mathrm{H}} r} \frac{\partial}{\partial r} r h n_{\mathrm{H}} D \frac{\partial c_{i}^{(\mathrm{s})}}{\partial r}+\frac{R_{i}}{n_{\mathrm{H}}} .
$$

Here $v_{r}$ is the radial drift velocity, $D$ the diffusion coefficient by turbulence, $R_{i}$ are the reaction rates per unit volume and time, $h$ is the disc height, and $n_{\mathrm{H}}$ is the number density of $\mathrm{H}$ nuclei for which we have the continuity equation for the total particle density

$$
\frac{\mathrm{d} n_{\mathrm{H}}}{\mathrm{d} t}=\frac{\partial n_{\mathrm{H}}}{\partial t}+\frac{1}{h r} \frac{\partial h r n_{\mathrm{H}} v_{r}}{\partial r}=0 .
$$

In writing down the transport-reactions equations in the form given by Eq. (2), we have assumed that mixing processes within the disc by small-scale turbulence-like flows associated with the process driving disc accretion always occur on much shorter timescales than radial mixing, such that the concentrations $c_{i}^{(\mathrm{s})}$ do not vary substantially in vertical direction. This assumption is satisfied, as can be seen from Fig. 3, where the characteristic timescales

$\tau_{\text {hor }}=r^{2} / D, \quad \tau_{\text {vert }}=h^{2} / D$

for diffusive mixing in horizontal and vertical direction are shown for the inner part of the solar nebula. Additionally, it is assumed that chemical processing of the carbonaceous material in the unshielded outermost disc layers by photo-processes is unimportant ${ }^{1}$. Such processes are currently not included in our model, but they may form an additional route to carbon oxidation (see Lee et al. 2010). Therefore we assume that $c_{i}^{(\mathrm{s})}(r, t)$ represents a vertically averaged concentration of the carbonaceous component $i$. Additionally, it is assumed that there is no substantial relative motion between dust and carrier gas.

The set of Eqs. (2) has to be solved with appropriate initial and boundary conditions. The initial condition is that we start with concentrations equal to the initial mixture that most likely originated from the parent molecular cloud,

$c_{i}^{(\mathrm{s})}(r, 0)=c_{0, i}^{(\mathrm{s})}$.

The same concentrations are prescribed at the outer disc radius,

$c_{i}^{(\mathrm{s})}\left(r_{\mathrm{ext}}, t\right)=c_{0, i}^{(\mathrm{s})}$.

1 Generally, carbonaceous material could be easily processed by photo-processes as has been shown in the work of Alata et al. (2014, 2015), for example. In proto-planetary disks, such processes would be only active in the disc atmosphere, which is not shielded from the ionizing radiation from the proto-star, but contains only a small fraction of the total mass. The relative importance of such surface processes in comparison to the processes that operate in the disks interior and affect the overwhelming majority of the material requires $2 \mathrm{D}$ disc models that include vertical mixing in the chemistry calculation. Such an extension of the model calculation is beyond the scope of the present paper. 

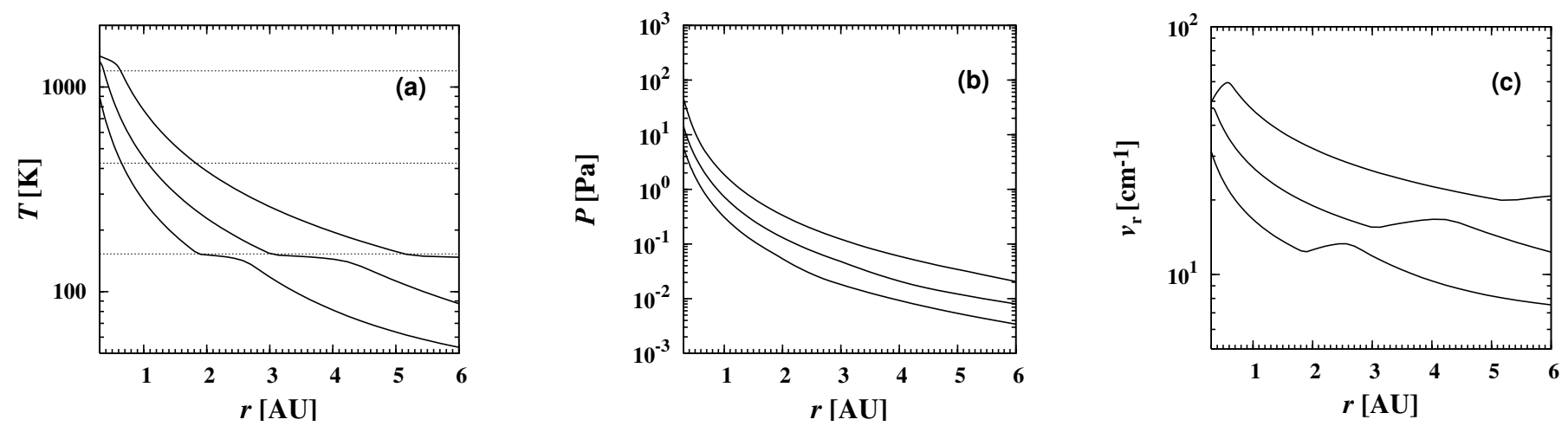

Fig. 2. Radial variation of some disc properties at three different ages of $1 \mathrm{Ma}, 2 \mathrm{Ma}$, and $3 \mathrm{Ma}$ (from top to bottom). Panel a: temperature at the mid-plane. The dotted horizontal lines correspond to the vaporisation of ice $(152 \mathrm{~K})$, pyrolysis of kerogen $(450 \mathrm{~K})$, and oxidation of soot $(\sim 1200 \mathrm{~K})$. Panel $b$ : pressure at the mid-plane. Panel $c$ : radial accretion velocity.

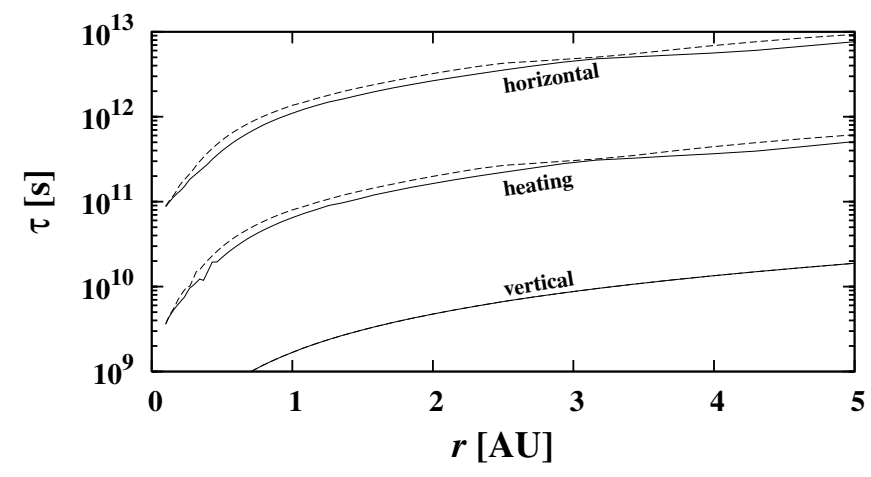

Fig. 3. Characteristic heating timescales in the inner disc region for a solar nebula model (from Sect. 6.1) at disc ages of $1 \mathrm{Ma}$ (solid line) and $2 \mathrm{Ma}$ (dashed line). Shown are the mixing timescales in horizontal and vertical direction, $\tau_{\text {hor }}$ and $\tau_{\text {vert }}$, respectively, and the timescale for heating of disc material, $\tau_{\text {heat }}$, due to inward drift by accretion.

The inner radius of the disc model is close to the radius of the proto-sun at a distance where all condensed phases are destroyed. Hence we prescribe

$c_{i}^{(\mathrm{s})}\left(r_{\mathrm{in}}, t\right)=0$

at the inner radius.

The details for evaluating the reaction rates $R_{i}$ of the pyrolysis processes are described in Appendix A.

\section{Carbon abundance in the quiet solar nebula}

The high temperatures required to destroy the carbonaceous component of the pristine disc material are achieved either by moving the material into the hot inner zones of the solar nebula by the accretion flow, or by the flash-like heating events that resulted in chondrule formation. In this section we consider the first process, the gradual heating by accretion; flash heating is considered in the next section.

\subsection{Disc model}

We study the distribution of the carbonaceous material in the quiet solar nebula, that is, without chondrule formation events, on the basis of a simple one-zone vertically averaged timedependent model of an accretion disc around a solar-like star. From such models we use the radial variation of temperature and
Table 2. Parameters of the one-zone disc model.

\begin{tabular}{llll}
\hline \hline Quantity & Symbol & Value & Unit \\
\hline Inner radius & $r_{\text {in }}$ & 0.1 & $\mathrm{AU}$ \\
Outer radius & $r_{\text {ext }}$ & 200 & $\mathrm{AU}$ \\
Stellar mass & $M_{*}$ & 0.95 & $M_{\odot}$ \\
disc mass & $M_{\text {disc }}$ & 0.05 & $M_{\odot}$ \\
Angular momentum & $J$ & $1.6 \times 10^{52}$ & $\mathrm{~g} \mathrm{~cm}^{2} \mathrm{~s}^{-1}$ \\
Viscosity parameter & $\alpha$ & $3 \times 10^{-3}$ & \\
\hline
\end{tabular}

particle density in the disc. This allows us to model the chemical processes acting near the mid-plane of the disc where temperatures and densities are highest in an actively accreting disc and where the chemistry is dominated by neutral-neutral reactions. Processes acting at the disc surface (discussed e.g. by Lee et al. 2010), where the material is subject to ionizing radiation that drives an ion-molecule reaction chemistry, cannot be modeled this way. This requires at least 2D disc models, which are beyond the scope of the present paper.

A disc model was calculated for the evolution of the solar nebula. The solution of the model was coupled with a solution of a set of chemical reaction and transport equations, Eq. (2), which allowed simultaneously modelling the processing of dust by chemical processes and the mixing of dust material between different disc zones by turbulent flows that are associated with the viscous process that drives the disc accretion. The model includes the dust components discussed in Gail (2004) and the pyrolysis and oxydation of the carbonaceous material. Because the carbonaceous material forms a significant contribution to the opacity of the disc material, the gasification of this component of the condensed matter lowers the opacity and by this, backreacts on the temperature structure of the accretion disc and its evolution. For this reason, a consistent coupling between disc evolution model and chemistry is necessary and is included in our model. The details of the model calculation are described in Gail (2001) and Wehrstedt \& Gail (2002) and are not repeated here.

The disc model was evolved from some initial state over a time interval of three million years, which covers the essential period during which planetesimals are likely to form. The model parameters used for construction of the disc model are shown in Table 2. The model used $\alpha$-viscosity as parameterisation of the process that drives disc accretion. The initial model was constructed such that the region between 0.1 and $30 \mathrm{AU}$ contained 
Table 3. Element abundances of the solar system, as used in the model calculations (given in the astronomical scale $A(\mathrm{El})=\log (n(\mathrm{El}) / n(\mathrm{H}))$ 12).

\begin{tabular}{lcllll}
\hline \hline Element & Abund. & Ref. & Element & Abund. & Ref. \\
\hline $\mathrm{He}$ & 10.98 & 1 & $\mathrm{Mg}$ & 7.53 & 2 \\
$\mathrm{C}$ & 8.47 & 1 & $\mathrm{Al}$ & 6.43 & 2 \\
$\mathrm{~N}$ & 7.87 & 1 & $\mathrm{Si}$ & 7.51 & 2 \\
$\mathrm{O}$ & 8.73 & 1 & $\mathrm{Fe}$ & 7.45 & 2 \\
$\mathrm{Z}$ & 0.142 & & & & \\
\hline
\end{tabular}

References. (1) Asplund et al. (2009); (2) Lodders et al. (2009).

a mass $M_{\text {disc }}$ (the total disc mass is higher, but only the mass in this part of the disc is relevant for our problem), and an angular momentum $J$ (assumed to be ten times the current angular momentum carried by present-day planets).

The element abundances used for the model calculation are given in Table 3 . They are required for the opacity calculation, which considers the most important mineral components and the carbonaceous material.

Figures $2 \mathrm{a}$ and $\mathrm{b}$ show the radial variation of temperature and pressure in the mid-plane of the disc for the range of distances where the terrestrial planets and bodies from the asteroid belt are located. The results are shown for evolutionary stages of the disc corresponding to ages of 1, 2, and $3 \mathrm{Ma}$, which cover the range of possible formation ages of the bodies in the inner solar system. Figure $2 \mathrm{c}$ shows the accretion velocity, which determines how disc matter is transported into regions of increasing temperature. This inward flow of matter rules the thermal metamorphism of the carbonaceous material, which is what we intend to study. The mixing timescales by turbulent diffusion and the characteristic timescale for temperature increase by accretion that result from the disc model are shown in Fig. 3.

\subsection{Radial variation of carbon abundance}

Figure 4a shows the result for the abundance variation of the different components of the carbonaceous material (including amorphous soot) with temperature in the mid-plane of the disc for $t=0.2,0.5,1$, and $2 \mathrm{Ma}$ after disc formation. The pyrolysis process depends mainly on the activation energy and only to a minor extent on the rate of temperature increase (see Eq. (A.2)) as the matter spirals-in into regions of higher temperature during disc accretion. For this reason, the variation over time of the fractional abundance curves with temperature for each of the materials are moderate to small. It is easy to recognize in which way the different materials disappear at their characteristic pyrolysis temperatures.

The dots with error bars show the temperature in the zone from which the planets acquire most of their material, according to the model of Lewis (1974). This considers the varying composition of solids in equilibrium with each other, and the gas phase with varying temperature (and pressure) and compares this with the composition of the planets. Although the assumptions on which this model resides are now strongly questioned, it may still serve as an indication of the relevant temperature range of in the solar nebula from which the planets assembled the greater part of their material. The model calculation shows that in the main feeding zones of the terrestrial planets, the volatile carbonaceous components are lost to the gas phase by pyrolysis,
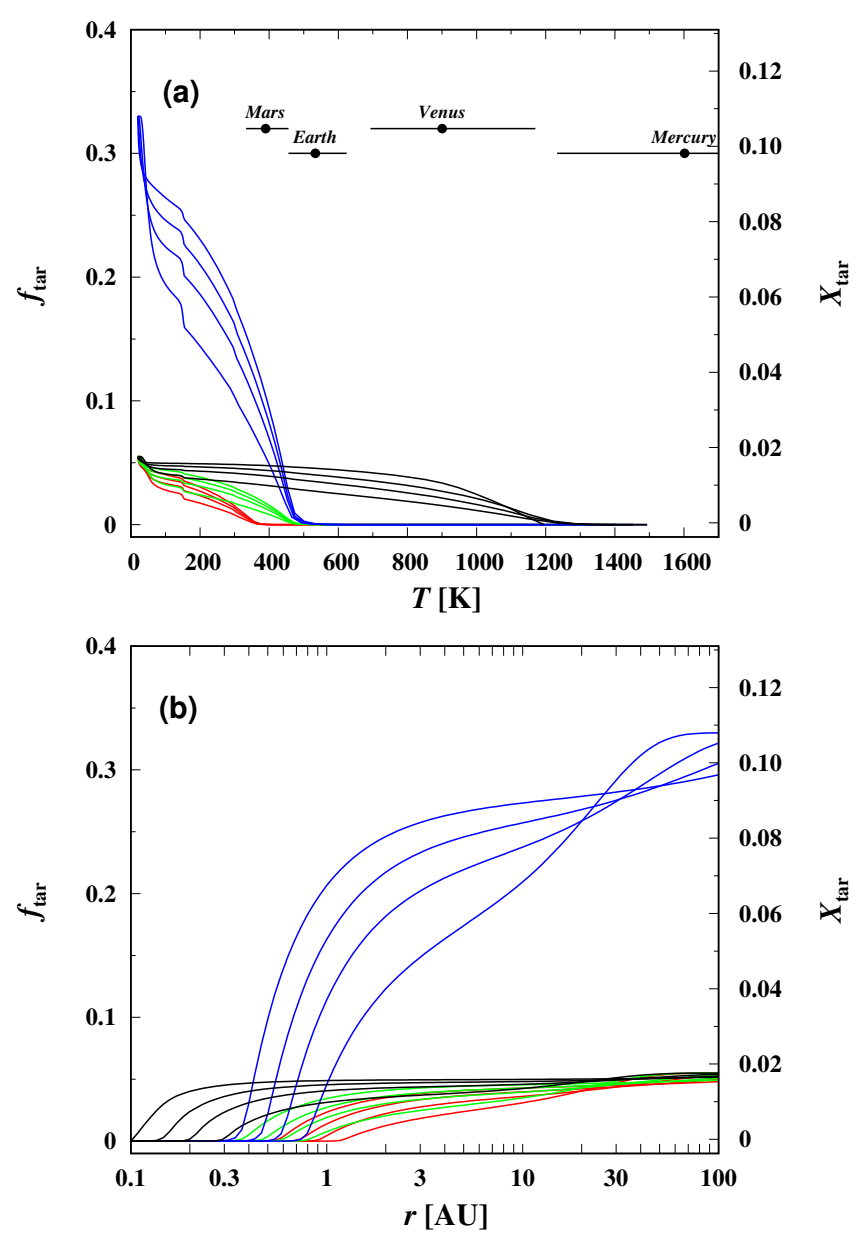

Fig. 4. Panel $a$ : variation with temperature of the fractions (left scale) of the carbon that is bound into one of the solid carbonaceous phase at the mid-plane of the disc model after 0.2, 0.5, 1, and $2 \mathrm{Ma}$ of disc evolution (from the lower to the upper curves in each group, respectively). Black lines show amorphous carbon. Blue lines depict refractory organic material $(\mathrm{CHON})$. Moderately volatile organics are delineated with green lines, and volatile organic material with red lines. The black dots with a horizontal bar indicate the possible range of temperatures in the feeding zones, which mainly contribute material to the planets. The right scale shows the mass fraction of carbon contained in dust grains. Panel $b$ : radial variation of the fractions of all components of the carbonaceous material at the mid-plane of the disc model after 0.2, 0.5, 1, and $2 \mathrm{Ma}$ of disc evolution (lower to upper curves for $r<10 \mathrm{AU}$ ).

but that the amorphous carbon component fully contributes the material that forms the planetesimals in the formation zone of Mars to Venus. Only the formation zone of Mercury would be carbon free as a result of oxidation.

Figure $4 \mathrm{~b}$ shows the variation of the fractional abundances with radius for the same four evolutionary periods. In this representation the evolutionary effects are clearly visible because the disc looses matter to the star and gradually becomes cooler as the surface density decreases. This shifts all the abundance curves inward. The curves remain essentially self-similar, however, such that the different disc evolutionary stages appear almost identical in the representation in Fig. 4a.

From these figures we infer that the resulting carbon content of the planetesimals in the formation zone of Mars to Venus would correspond to a mass fraction of serveral percent. This strongly exceeds the carbon content estimated in models for the mantle composition of terrestrial planets. Furthermore, the 


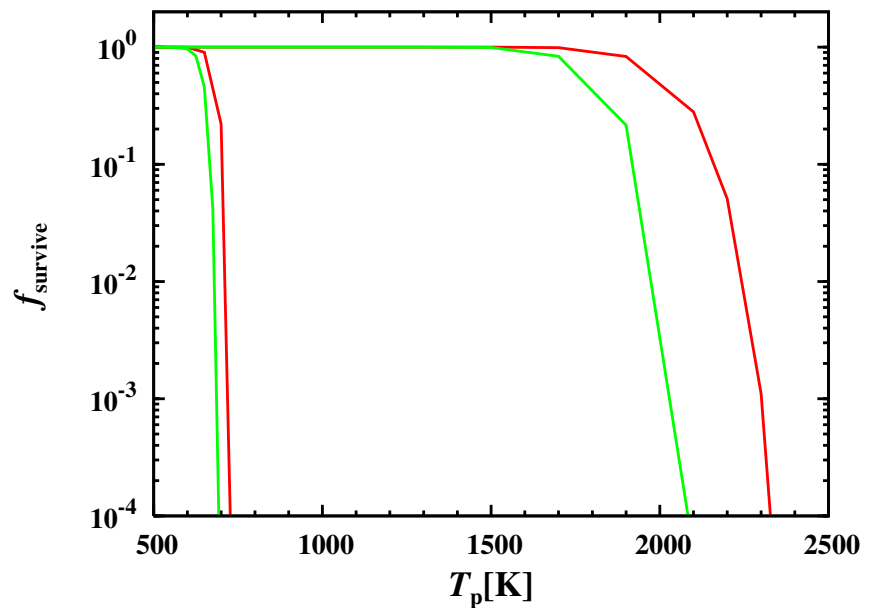

Fig. 5. Fraction of refractory anthracene (left) and amorphous carbon (right) that survives flash-heating during chondrule formation for different cooling times: $\lambda^{-1}=10 \mathrm{~h}$ (red lines) and $\lambda^{-1}=100 \mathrm{~h}$ (green lines).

carbon content of ordinary chondrites, for example, which are assumed to originate in the region $\lesssim 2.5 \mathrm{AU}$, would exceed $10 \%$, which also contradicts observations. The low carbon content of the terrestrial planets and ordinary chondrites therefore cannot be explained by gradual warming and associated decomposition and chemical reduction to $\mathrm{CO}$ during accretion flow. It requires a different process.

\section{Carbon destruction by flash-heating}

Except for the CI chondrites, all chondrite classes contain chondrules that are thought to be the result of flash-like heating processes acting in the solar nebula. Despite considerable effort in cosmochemistry, the process or processes responsible for their formation remains unclear, but these investigations provide much information on the thermal history of chondrules derived from the findings with respect to their composition and texture. These investigations showed (see Jones et al. 2000; Ciesla 2005, for reviews) that the chondrules were heated to high temperatures with peak values in the range between about 1600 and 2100 $\mathrm{K}$. The main facts are as follows. (1) The chondrules are heated very rapidly and remain for only a brief period of a few minutes at their peak temperature. (2) They start heating from low temperatures. (3) They cool down after the flash-like heating at rates of $10 \mathrm{~K} / \mathrm{h}$ to $1000 \mathrm{~K} / \mathrm{h}$. (4) Chondrule formation events occur repeatedly.

It is to be expected that the temperature evolution reconstructed for the chondrules also reflects that of the ambient gas. The carbonaceous component of any dust aggregate initially present in the disc would start to decompose and oxidise (Appendix C) once it experiences a flash-heating event. The important question then is whether the duration of the hightemperature spikes responsible for chondrule formation is sufficiently long for carbon to be completely destroyed, or whether part of it survives such events. To determine this, we solved the system of rate Eqs. (2) without mixing and transport terms (these are negligible over the short duration of a flash event) for a fixed mass density corresponding to a pressure of $10^{-4}$ bar at $300 \mathrm{~K}$. This pressure is typical for the region around $2 \mathrm{AU}$ in the solar nebula (see Fig. 2b) where the chondrite parent bodies formed. The temperature was varied according to the following prescription:

1. The temperature is increased linearly within $1 \mathrm{~min}$ from $300 \mathrm{~K}$ to some maximum temperature $T_{\mathrm{p}}$.

2. The maximum (plateau) temperature $T_{\mathrm{p}}$ is held fixed for $10 \mathrm{~min}$.

3. The temperature decreases exponentially from $T_{\mathrm{p}}$ with decay constant $\lambda$ of several hours.

This was taken as an approximation to the temperature spikes that are responsible for chondrule formation. The plateau temperature $T_{\mathrm{p}}$ was varied between $1600 \mathrm{~K}$ and $2100 \mathrm{~K}$, as is suggested by the results of investigations on chondrules. The initial conditions for the gas phase and the carbonaceous components are described in Appendix C. The reaction network is supplemented in this case by the reaction

$\mathrm{C}_{2}[\mathrm{~s}]+\mathrm{O} \longrightarrow \mathrm{C}_{2} \mathrm{O}$

because in contrast to the quiet disc, the peak temperature during chondrule-forming flash-heating is sufficiently high for $\mathrm{O}$ atoms to become abundant gas-phase species that contribute to carbon oxidation.

Figure 5 shows the fractions of the initial content that survive a single flash event for two of the carbonaceous components, amorphous carbon and anthracene from the refractory $\mathrm{CHON}$ component, for varying plateau temperatures and different decay times $\lambda^{-1}$ after passing the peak temperature. The amorphous carbon is rather resistant. Only flash-heating events with the highest peak temperatures destroy the amorphous carbon. The next refractory of the carbonaceous components, the refractory anthracene from the CHON material, would only survive heating events with temperatures lower than $700 \mathrm{~K}$. Hence, this and all other less strongly refractory components of the pristine carbonaceous material are completely destroyed by the flashheating events that occur in the solar nebula, as documented by the existence and properties of chondrules.

\section{Carbon content of planetesimals}

From our calculations it follows that the pristine carbonaceous component of the dust material in the quiet disc that is transported inwards during the accretion process is gasified in the warm inner parts of the solar nebula. The volatile and refractory hydrocarbons disappear between $300 \mathrm{~K}$ and $500 \mathrm{~K}$ by pyrolysis, while the minor component, the amorphous carbon, survives up to about $1200 \mathrm{~K}$ until this also disappears by oxidation with water vapour. When the planetesimal formation process commences in the solar nebula, the planetesimals would initially be endowed with significant amounts of solid carbonaceous material between several mass percents in the inner terrestrial planet region and up to more than about 10 mass percent in the asteroidal region. This is much more than what is actually found in their present-day descendants.

The second important process that results in efficient destruction of carbonaceous material is the process that results in the formation of chondrules. The hydrocarbon components of the carbonaceous components of the pristine dust material are all gasified during flash-heating, except for the amorphous carbon. The amorphous carbon is only partially destroyed in a single such event, and this occurs only for the flash-heating events that reach the highest peak temperatures inferred from chondrule properties. Investigations of chondrules revealed that the flashheating is a repeated process because many chondrules show 


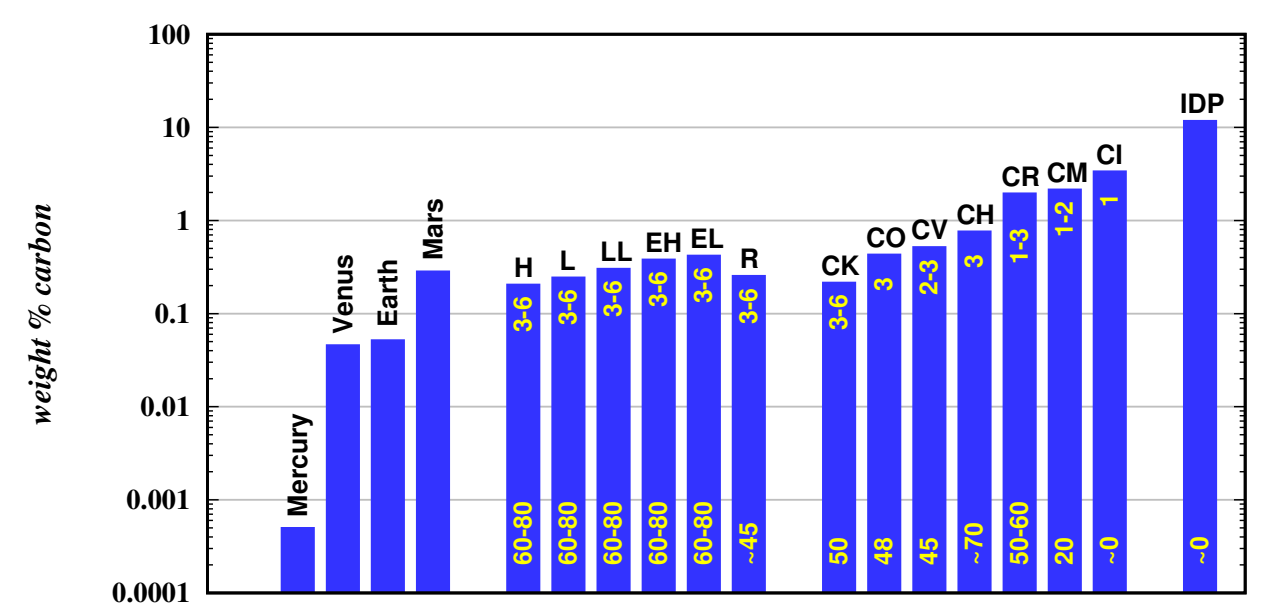

Fig. 6. Carbon content of bodies in the terrestrial planet region, in the asteroid belt, and in cometary particles (anhydrous porous interplanetary dust particles). The lower numbers in the bars give the percentage of volume filled by chondrules, and the upper numbers show the range of petrologic types. For the sources of the data, see text. indications that they contain material from former chondruleforming episodes (e.g. Jones et al. 2000). In the regions of the solar nebula where this process was active, the carbon content of the dust will be strongly reduced to values below the small initial fraction of amorphous carbon, or the dust material is even more or less completely cleared from carbon.

About $80 \%$ of the parent bodies of ordinary chondrites were composed of chondrules and contained only a small residual fraction of dust (=matrix). The disc material in this region unavoidably must have been subject to many repeated flash-heating events, which means that the initial carbon content must have been nearly completely destroyed. This does not mean that this region is completely cleared from carbon, however, because some matrix material may have escaped flash heating events, and/or the extended chondrule formation processes may have extended over hundreds of thousands of years, which leaves enough time to mix some fresh carbon-bearing material into this region from outside. Hence, we expect a small but non-zero carbon content of the disc material in this region at the instant when the solid material is assembled into planetesimals.

A quantitative statement on the resulting carbon content cannot be made at present. A model calculation referring to this requires knowing the frequency and exact intensity of the flashheating events. These parameters are not known at present.

A different situation is obviously encountered in the disc region beyond the ice-line, where the carbonaceous chondrites formed. The CI chondrites are practically chondrule free, and the CM chondrites contain only a low fraction of chondrules. This means that the process responsible for the flash-heating events that formed chondrules did not work in this region, or it was rather inefficient, such that flashes are rare, but individual flashes are still sufficiently energetic to produce typical chondrule textures.

That chondrule flash-heating events and chondrule abundances control the carbon content of chondrites and their parent bodies is immediately supported by the observation that chondrules are highly depleted in carbon, while the matrix component is the carbon carrier, particularly in carbonaceous chondrites (e.g. Makjanic et al. 1993; Alexander et al. 2007; Pearson et al. 2006).

These general trends are seen in the different meteorite classes. Figure 6 shows the carbon content of the different chondrite classes and that of anhydrous IDPs, which are derived from comets. For the different chondrite classes, the volume fraction of chondrules is also noted. The compilation of Henke et al. (2016, Table 1, based on the sources cited therein) for the chondrites and from Morgan \& Anders (1979, 1980), Marty (2012), and McDonough \& Sun (1995) for the planets are listed. The carbon abundances are adopted from Jarosewich (1990) and Pearson et al. (2006). The chondrule abundance of CK chondrites is given in the literature as $15 \%$, but a new determination by Chaumard \& Devouard (2016) found $~ 50$ volume percent for CK3 meteorites. It has been proposed that CK and CV are derived from the same parent body (Greenwood et al. 2010b), and this may be the parent body of the Eos family (Greenwood et al. 2010a; Novaković \& Tsirvoulis 2014). The chondrule abundance for $\mathrm{CH}$ chondrites is taken from Hezel et al. (2001).

There clearly is some general trend of decreasing chondrule abundance with increasing carbon content, which fits the expectations following from the carbon gasification and flash-heating processes acting in the solar nebula. Specifically, the following groups can be distinguished.

1. IDPs. Their carbon content represents the initial state that is found at distances $\gtrsim 10 \mathrm{AU}$ where the comets formed. Chondrule fragments occasionally found in IDPs are thought to be formed in the warm parts of the solar nebula and transported outwards (e.g. Brownlee 2014).

2. The CI and CM chondrites, where chondrules have low abundance or are almost absent. Their high water content indicates that they are formed in the trans-snow-line region of the solar nebula. Their carbon abundance is lower than in IDPs, which can partly be ascribed to their formation at different disc radii and processes related to their parent bodies.

3. The $\mathrm{CV}+\mathrm{CK}, \mathrm{CO}$, and the $\mathrm{R}$ chondrites, where chondrules are abundant but not dominant and for which the carbon content is significantly lower. Their low water content indicates that they were formed not too far away from but inside of the snow-line region of the solar nebula.

4. The ordinary chondrites, with very abundant chondrules, absence of water, and a somewhat smaller carbon abundance than the previous group. They are obviously formed in a region where repeated flash-heating events destroy the carbon that is carried inwards by the accretion process.

5. The terrestrial planets with a significantly lower carbon content than chondrites. They form in a region where only the small fraction of amorphous carbon could survive in the quiescent nebula, but almost all of this is likely to be previously destroyed by flash-heating since mass accretion in the disc requires the material first to pass through the region $r \gtrsim 2 \mathrm{AU}$ before it enters the terrestrial planet-forming region. The small surviving fraction of the carbon would be included in the planetesimals and the planets formed from 
them. During the differentiation into core and mantle, this carbon could partly be dissolved in the core material, leaving a mantle depleted in carbon. This is derived from recent studies that found that the outer core of Earth may contain some carbon at the 1\% weight level (Nakajima et al. 2015; Wood et al. 2013).

6. Mercury, which formed in a region of the solar nebula where the resilient amorphous carbon is also destroyed by oxidation, such that no condensed carbon exists in this region.

However, there are also some obvious discrepancies that indicate other or additional processes might govern the carbon content.

1. The $\mathrm{CR}$ and $\mathrm{CH}$ chondrites with their high chondrule content do not easily fit into this picture. They belong to a common meteorite clan formed by $\mathrm{CR}, \mathrm{CH}$, and $\mathrm{CB}$ (Krot et al. 2014). The CB chondrites are not considered here because they are probably formed as the product of an asteroidal collision (Fedkin et al. 2015). The same seems to hold for $\mathrm{CHs}$ as well (Krot et al. 2010), such that their properties are not solely determined by solar nebula processes.

2. Loss of carbon on parent bodies due to high-temperature metamorphism. For example, Alexander et al. (2007) found that in thermally metamorphosed type 3 ordinary and CV chondrites, carbon could be depleted by a factor of 2-3, similar to earlier work by Makjanic et al. (1993). Similarly, differentiation that might have affected the precursor planetesimals of terrestrial planets could also have caused significant carbon loss.

3. Reduced assemblages generally have higher carbon contents, for instance, enstatite chondrites, when compared to ordinary chondrites, or $\mathrm{CH}$ when compared to $\mathrm{CO}$ chondrites.

\section{Concluding remarks}

We modelled the processing of carbonaceous matter in the solar proto-planetary disc. Much of the carbon originally contained in solid phases is gasified and lost and cannot be incorporated into planetary bodies, particularly those forming in the inner disc. However, the carbon depletion of ordinary chondrites and terrestrial planets is higher than expected from combustion at typically modelled disc temperatures. Chondrule flash-heating is an effective additional process causing carbon depletion in chondritic planetesimals, which result is corroborated by the fundamental observation that the main carbon carrier in chondritic meteorites is the fine-grained matrix, while chondrules are generally poor in carbon.

While it is not completely ascertained to which extent the precursor material of the terrestrial planets contained chondrules, the ubiquity of chondrules in undifferentiated planetesimals indicates that chondrule-forming processes were active throughout the solar nebula, particularly in the inner disc. While the incorporation of carbon-poor chondrules into precursor planetesimals is an essential factor of carbon depletion, an additional reduction of the carbon content may have been due to thermal processing on planetesimals due to heating by ${ }^{26} \mathrm{Al}$ decay heat, which led to strong thermal metamorphism or even differentiation into metallic cores and silicate mantles of even relatively small (about $100 \mathrm{~km}$ sized) planetesimals.

Acknowledgements. This work was supported by "Schwerpunktprogramm 1385" supported by the Deutsche Forschungsgemeinschaft (DFG). M.T. acknowledges support by the Klaus Tschira Stiftung gGmbH. This research has made use of NASA's Astrophysics Data System.

\section{References}

Alata, I., Cruz-Diaz, G. A., Muñoz Caro, G. M., \& Dartois, E. 2014, A\&A, 569, A119

Alata, I., Jallat, A., Gavilan, L., et al. 2015, A\&A, 584, A123

Alexander, C. M. O. 2011, in Molecular Universe, eds. J. Cernichario, \& R. Bachiller, IAU Symp., 280, 288

Alexander, C. M. O., Fogel, M., Yabuta, H., \& Cody, G. D. 2007, Geochim. Cosmochim. Acta, 71, 4380

Appel, J., Bockhorn, H., \& Frenklach, M. 2000, Combustion \& Flame, 121, 122 Asplund, M., Grevesse, N., Sauval, A. J., \& Scott, P. 2009, ARA\&A, 47, 481

Bergin, E. A., Blake, G. A., Ciesla, F., Hirschmann, M. M., \& Li, J. 2015, Proceedings of the National Academy of Science, 112, 8965

Bradley, J. P. 1994, Geochim. Cosmochim. Acta, 58, 2123

Brownlee, D. 2014, Ann. Rev. Earth Planet. Sci., 42, 179

Brownlee, D., Tsou, P., Aléon, J., et al. 2006, Science, 314, 1711

Burnham, A. K., Braun, R. L., Gregg, H. R., \& Samoun, A. M. 1987, Energy and Fuels, 1, 452

Chaumard, N., \& Devouard, B. 2016, Meteor. Planet. Sci., 51, 547

Chyba, C. F., Thomas, P. J., Brookshaw, L., \& Sagan, C. 1990, Science, 249, 366

Ciesla, F. J. 2005, in Chondrites and the Protoplanetary Disk, eds. A. N. Krot, E. R. D. Scott, \& B. Reipurth, ASP Conf. Ser., 341, 811

Clairemidi, J., Bréchignac, P., Moreels, G., \& Pautet, D. 2004, Planet. Space Sci., 52,761

Clairemidi, J., Moreels, G., Mousis, O., \& Bréchignac, P. 2008, A\&A, 492, 245

Clemett, S. J., Sandford, S. A., Nakamura-Messenger, K., Hörz, F., \& McKay, D. S. 2010, Meteor. Planet. Sci., 45, 701

Cottin, H., \& Fray, N. 2008, Space Sci. Rev., 138, 179

Da Rio, N., Jeffries, R. D., Manara, C. F., \& Robberto, M. 2014, MNRAS, 439, 3308

Derenne, S., \& Robert, F. 2010, Meteor. Planet. Sci., 45, 1461

Duschl, W. J., Gail, H.-P., \& Tscharnuter, W. M. 1996, A\&A, 312, 624

Eberhardt, P., Krankowsky, D., Schulte, W., et al. 1987, A\&A, 187, 481

Ehrenfreund, P., Charnley, S. B., \& Wooden, D. 2004, in Comets II, eds. M. C. Festou, H. U. Keller, \& H. A. Weaver (Tucson: University of Arizona Press), 115

Fedkin, A. V., Grossman, L., Humayun, M., Simon, S. B., \& Campbell, A. J. 2015, Geochim. Cosmochim. Acta, 164, 236

Finocchi, F., Gail, H.-P., \& Duschl, W. J. 1997, A\&A, 325, 1264

Flynn, G. J., \& Keller, L. P. 2003, in Workshop on Cometary Dust in Astrophysics, eds. D. E. Brownlee, L. P. Keller, \& S. R. Messenger, 6007

Flynn, G. J., Bajt, S., Sutton, S. R., et al. 1996, in Physics, Chemistry, and Dynamics of Interplanetary Dust, eds. B. A. S. Gustafson, \& M. S. Hanner, IAU Colloq., 150, ASP Conf. Ser., 104, 291

Flynn, G. J., Keller, L. P., Feser, M., Wirick, S., \& Jacobsen, C. 2003, Geochim. Cosmochim. Acta, 67, 4791

Flynn, G. J., Keller, L. P., Jacobsen, C., \& Wirick, S. 2004, Adv. Space Res., 33, 57

Flynn, G. J., Keller, L. P., Wirick, S., \& Jacobsen, C. 2008, in Organic Matter in Space, eds. S. Kwok, \& S. Sandford (Cambridge University Press), IAU Symp., 251, 267

Flynn, G. J., Wirick, S., \& Keller, L. P. 2013, Earth Planets Space, 65, 1159

Fomenkova, M. N. 1997, in From Stardust to Planetesimals, ed. Y. J. Pendleton, ASP Conf. Ser., 122, 415

Fomenkova, M. N. 1999, Space Sci. Rev., 90, 109

Fomenkova, M. N., \& Chang, S. 1994, in Analysis of Interplanetary Dust Particles, ed. M. E. Zolensky, 22

Fomenkova, M. N., Chang, S., \& Mukhin, L. M. 1994, Geochim. Cosmochim. Acta, 58, 4503

Gail, H.-P. 2001, A\&A, 378, 192

Gail, H.-P. 2002, A\&A, 390, 253

Gail, H.-P. 2004, A\&A, 413, 571

Geiss, J. 1987, A\&A, 187, 859

Grady, M. M., \& Wright, I. P. 2003, Space Sci. Rev., 106, 231

Greenwood, R. C., Franchi, I. A., Chaumard, N., Devouard, B., \& Burbine, T. H. 2010a, Meteor. Planet. Sci. Suppl., 73, 5430

Greenwood, R. C., Franchi, I. A., Kearsley, A. T., \& Alard, O. 2010b, Geochim. Cosmochim. Acta, 74, 1684

Henke, S., Gail, H.-P., Trieloff, M., Schwarz, W. H., \& Kleine, T. 2012, A\&A, 537, A45

Henke, S., Gail, H.-P., \& Trieloff, M. 2016, A\&A, 589, A41

Henning, T., \& Semenov, D. 2013, Chem. Rev., 113, 9016

Hezel, D. C., Brenker, F. E., \& Palme, H. 2001, Meteor. Planet. Sci. Suppl., 36, A80

Jarosewich, E. 1990, Meteoritics, 25, 323 
Jessberger, E. K. 1999, Space Sci. Rev., 90, 91

Jessberger, E. K., Kissel, J., \& Rahe, J. 1989, in Origin and Evolution of Planetary and Satellite Atmospheres, eds. S. K. Atreya, J. B. Pollack, \& M. S. Matthews (Tucson: University of Arizona Press), 167

Jones, A. P. 2016, Roy. Soc. Open Sci., 3, 160221

Jones, R. H., Lee, T., Connolly, Jr., H. C., Love, S. G., \& Shang, H. 2000, Protostars and Planets IV, 927

Jones, A. P., Fanciullo, L., Köhler, M., et al. 2013, A\&A, 558, A62

Jones, A. P., Ysard, N., Köhler, M., et al. 2014, Faraday Discussions, 168, 313

Jones, A. P., Koehler, M., Ysard, N., Bocchio, M., \& Verstraete, L. 2017, A\&A, 602, A46

Keller, L. P., Messenger, S., \& Bradley, J. P. 2000, J. Geophys. Res., 105, 10397

Kissel, J., \& Krueger, F. R. 1987, Nature, 326, 755

Köhler, M., Ysard, N., \& Jones, A. P. 2015, A\&A, 579, A15

Krot, A. N., Nagashima, K., Yoshitake, M., \& Yurimoto, H. 2010, Geochim. Cosmochim. Acta, 74, 2190

Krot, A. N., Keil, K., Scott, E. R. D., Goodrich, C. A., \& Weisberg, M. K. 2014, in Meteorites and Cosmochemical Processes, ed. A. M. Davis (Elsevier), 1

Lawler, M. E., \& Brownlee, D. E. 1992, Nature, 359, 810

Lee, J.-E., Bergin, E. A., \& Nomura, H. 2010, ApJ, 710, L21

Lewis, J. S. 1974, Science, 186, 440

Li, A. 2009, in Deep Impact as a World Observatory Event: Synergies in Space, Time, and Wavelength, eds. H. U. Käufl, \& C. Sterken, 161

Lodders, K., Palme, H., \& Gail, H.-P. 2009, in Landolt Börnstein, New Series, Vol. VI/4B, ed. J.-E. Trümper (Berlin: Springer-Verlag), 560

Makjanic, J., Vis, R. D., Hovenier, J. W., \& Heymann, D. 1993, Meteoritics, 28, 63

Manara, C. F., Robberto, M., Da Rio, N., et al. 2012, ApJ, 755, 154

Marinov, N. M., Pitz, W. J., Westbrook, C. K., Castaldi, M. J., \& Senkan, S. M. 1996, Combust. Sci. Technology, 116, 211

Marty, B. 2012, Earth Planet. Sci. Lett., 313, 56

Mathis, J. S., Rumpl, W., \& Nordsieck, K. H. 1977, ApJ, 217, 425

McDonough, W. F., \& Sun, S.-S. 1995, Chem. Geol., 120, 223

McSween, H., \& Huss, G. R. 2010, Cosmochemistry (Cambridge: Cambridge University Press)
Messenger, S., Sandford, S., \& Brownlee, D. 2006, in Meteorites and the Early Solar System II, eds. D. S. Lauretta, \& H. Y. McSween (Tucson: University of Arizona Press), 187

Morgan, J. W., \& Anders, E. 1979, Geochim. Cosmochim. Acta, 43, 1601

Morgan, J. W., \& Anders, E. 1980, Proc. Natl. Acad. Sci. USA, 77, 6973

Mumma, M. J., \& Charnley, S. B. 2011, ARA\&A, 49, 471

Nakajima, Y., Imada, S., Hirose, K., et al. 2015, Nature Communications, 6, 8942

Nakano, H., Kouchi, A., Tachibana, S., \& Tsuchiyama, A. 2003, ApJ, 592, 1252 Novaković, B., \& Tsirvoulis, G. 2014, in Asteroids, Comets, Meteors 2014, Proc. Conf., eds. K. Muinonen, A. Penttilä, M. Granvik, et al.

Oba, M. 2002, Ph.D. Thesis, University of Tsukuba

Pearson, V. K., Sephton, M. A., Franchi, I. A., Gibson, J. M., \& Gilmour, I. 2006, Meteor. Planet. Sci., 41, 1899

Pollack, J. B., Hollenbach, D., Beckwith, S., Simonelli, D. P., \& Fong, W. 1994, ApJ, 421, 615

Scott, P., Grevesse, N., Asplund, M., et al. 2015, A\&A, 573, A25

Thomas, K. L., Keller, L. P., Blanford, G. E., \& McKay, D. S. 1993, Lun. Planet. Sci. Conf., 24

Thomas, K. L., Keller, L. P., Blanford, G. E., \& McKay, D. S. 1994, in Analysis of Interplanetary Dust Particles, ed. M. E. Zolensky, 49

Walsh, C., Herbst, E., Nomura, H., Millar, T. J., \& Weaver, S. W. 2014, Faraday Discuss., 168, 389

Wang, H., \& Frenklach, M. 1997, Combustion \& Flame, 110, 173

Wang, S., Liu, J., Jiang, X., Han, X., \& Tong, J. 2013, Oil Shales, 30, 27

Warnatz, J., Maas, U., \& Dibbe, R. W. 2006, Combustion (Berlin, Heidelberg: Springer)

Wehrstedt, M., \& Gail, H.-P. 2002, A\&A, 385, 181

Wooden, D. H. 2002, Earth, Moon, Planets, 89, 247

Wooden, D. H. 2008, Space Sci. Rev., 138, 75

Wood, B. J., Li, J., \& Shahar, A. 2013, Rev. Mineral. Geochem., 75, 231

Ysard, N., Köhler, M., Jones, A., et al. 2015, A\&A, 577, A110

Zolotov, M. Y., \& Shock, E. L. 2001, Icarus, 150, 323

Zubko, V., Dwek, E., \& Arendt, R. G. 2004, ApJS, 152, 211 
Table A.1. Data for pyrolysis of kerogen (from Oba 2002).

\begin{tabular}{lcccc}
\hline \hline Released species & $\begin{array}{c}A \\
{\left[\mathrm{~s}^{-1}\right]}\end{array}$ & $\begin{array}{c}E_{\mathrm{a}} \\
{[\mathrm{kJ} / \mathrm{mol}]}\end{array}$ & $\begin{array}{c}E_{\mathrm{a}} / R \\
{[\mathrm{~K}]}\end{array}$ & $\begin{array}{c}T_{\mathrm{py}} \\
{[\mathrm{K}]}\end{array}$ \\
\hline Benzene & $2.46 \times 10^{12}$ & 205.0 & 24650 & 450 \\
Hexane & $2.60 \times 10^{14}$ & 223.0 & 26810 & 450 \\
Indene & $4.90 \times 10^{11}$ & 195.4 & 23500 & 440 \\
\hline
\end{tabular}

\section{Appendix A: Rate coefficients for pyrolysis}

\section{A.1. Pyrolysis of carbonaceous material}

We here specify the reaction rates $R_{i}$ for the pyrolysis processes considered in Sect. 4.3. When we describe the species bound in one of the components of the carbonaceous material by a fictitious concentration $c_{i}^{(\mathrm{s})}$, and when we assume that the decomposition process can be described as a homogeneous first-order reaction process, then the reaction rate can be written as

$R_{i}=k_{i} c_{i}^{(\mathrm{s})} n_{\mathrm{H}}$

with the reaction rate coefficient $k_{i}$ in units per unit time.

We estimate the rate coefficient $k_{i}^{(\mathrm{c})}$ from results of laboratory experiments on pyrolysis of kerogen. The observed rates have been shown to be interpreted best of all by assuming that (1) the pyrolysis process is a homogeneous first-order rate process; and (2) that $k_{i}$ is described by a series of Arrhenius-law like terms

$k_{i}=A_{i} \mathrm{e}^{-E_{i, \mathrm{a}} / R T}$,

with activation energies $E_{i, \text { a }}$ from a certain range of values (Burnham et al. 1987). Because of the expected structural relationship of the carbonaceous material with kerogen, we can speculate that for the carbonaceous material of IDPs we also need such a description to correctly account for the liberation of pyrolysis products over an extended temperature range, which is also seen in the experiments of Nakano et al. (2003). On the other hand, only insufficient information is currently available to construct such refined models. We therefore approximated the rate $k_{i}$ by a single law of the form of Eq. (A.1).

In a study on kerogen pyrolysis, Oba (2002) obtained values for the prefactor $A$ and activation energy $E_{\mathrm{a}}$ for some important pyrolysis products. These are listed in Table A.1. Less specified data reported in Burnham et al. (1987) are similar. We calculate, as in Chyba et al. (1990), a release temperature from the relation

$T_{\text {py }}=\frac{E_{\mathrm{a}}}{R \ln \left(\tau_{\text {heat }} A\right)}$,

where $R$ is the gas constant and $\tau_{\text {heat }}$ is a characteristic heating time. At this temperature, the fraction $1 / \mathrm{e}$ is released by pyrolysis from the initial material if the material is held at this temperature for the period $\tau_{\text {heat }}$. Figure 3 shows the timescale for a substantial temperature change for a model of the solar nebula, defined here to be

$\tau_{\text {heat }}=0.1 \mathrm{r} / v_{\mathrm{r}}$

with $v_{r}$ being the inward drift velocity in an accretion disc. A typical value of $\tau_{\text {heat }}$ is $1 \ldots 3 \times 10^{11} \mathrm{~s}$ at distances in the range 1 ...3 AU. Typical release temperatures $T_{\text {py }}$ for some pyrolysis products calculated from this are shown in Table A.1.

The calculated release temperatures are comparable to the findings of laboratory experiments of Nakano et al. (2003) for
Table A.2. Kinetic data assumed for the pyrolysis of the carbonaceous material in the solar nebula.

\begin{tabular}{lccc}
\hline \hline Component & $\begin{array}{c}A \\
{\left[\mathrm{~s}^{-1}\right]}\end{array}$ & $\begin{array}{c}T_{0} \\
{[\mathrm{~K}]}\end{array}$ & $\mathrm{b}$ \\
& $4.00 \times 10^{13}$ & 19050 & \\
Volatile organic material & $4.00 \times 10^{13}$ & 24500 & \\
Moderately volatile organics & $4.00 \times 10^{13}$ & 24500 & \\
Refractory organic material & & & \\
$\mathrm{CH}_{4}[\mathrm{v}]$ & $2.00 \times 10^{13}$ & 19050 & \\
$\mathrm{C}_{4} \mathrm{H}_{10}[\mathrm{v}]$ & $5.00 \times 10^{12}$ & 19050 & \\
$\mathrm{C}_{16} \mathrm{H}_{10}[\mathrm{~m}]$ & $2.50 \times 10^{12}$ & 24500 & \\
$\mathrm{CH}_{4}[\mathrm{r}]$ & $6.00 \times 10^{12}$ & 24500 & \\
$\mathrm{CO}[\mathrm{r}]$ & $1.00 \times 10^{13}$ & 24500 & \\
$\mathrm{CO}_{2}[\mathrm{r}]$ & $8.00 \times 10^{12}$ & 24500 & \\
$\mathrm{C}_{16} \mathrm{H}_{10}[\mathrm{r}]$ & $1.00 \times 10^{12}$ & 24500 & \\
$\mathrm{C}_{2}[\mathrm{~s}]$ & $1.00 \times 10^{-15}$ & & 0.5 \\
\hline
\end{tabular}

their proxy for the cometary carbonaceous material, although the heating periods in the experiments were shorter, which means that the bond energies in the laboratory-made material are somewhat lower than for natural kerogen. It is to some extent a coincidence that the temperatures turn out to be about the same, despite different conditions in the experiment and the disc models.

We used the kerogen-based data in our model calculation for the pyrolysis of the CHON material. The rate term in Eq. (2) is

$\frac{R_{i}}{n_{\mathrm{H}}}=A_{i} \mathrm{e}^{-T_{0 i} / T} c_{i}^{(\mathrm{s})}$

for each of the components of the carbonaceous material (except soot) from Table 1. A single value for $A_{i}$ and $T_{0 i}$ was assumed for simplicity. The values of $T_{0 i}$ were slightly adjusted such that the pyrolysis temperatures given in Table 1 were reproduced. The rate coefficients for the loss of $\mathrm{C}$ atoms by pyrolysis for three components of the carbonaceous material are shown in the first three rows of Table A.2. The following row gives the coefficients for the pyrolysis rates of the individual products. They consider the relative fraction of carbon released by the different species, as given in Table 1, and the number of $\mathrm{C}$ atoms carried by them. It would be possible to account in more detail for the data of individual pyrolysis species given by Oba (2002), but in view of our only fragmentary present knowledge of the astronomical pyrolysis process, we currently cannot consider more details.

\section{A.2. Oxidation of soot}

The process of carbon particle oxidation has been extensively studied in flame chemistry. Duschl et al. (1996) and Finocchi et al. (1997) applied the results for the basic oxidation mechanism of solid carbon obtained in the chemistry of flames to the problem of carbon dust grain destruction in proto-planetary accretion discs. Under conditions encountered in the early solar nebula, the basic reaction scheme for oxidation of solid carbon into $\mathrm{CO}$ starts with the reaction where a $\mathrm{OH}$ radical attacks a six-ring at the periphery of a large PAH and cracks the carboncarbon bonds. The reaction is assumed to be of the type

soot $+\mathrm{OH} \longrightarrow \operatorname{soot}-2 \mathrm{C}+\mathrm{HCCO}$.

This can be cast into the form of a chemical reaction equation when we introduce an imagined species $C_{2}[s]$ that is bound in 

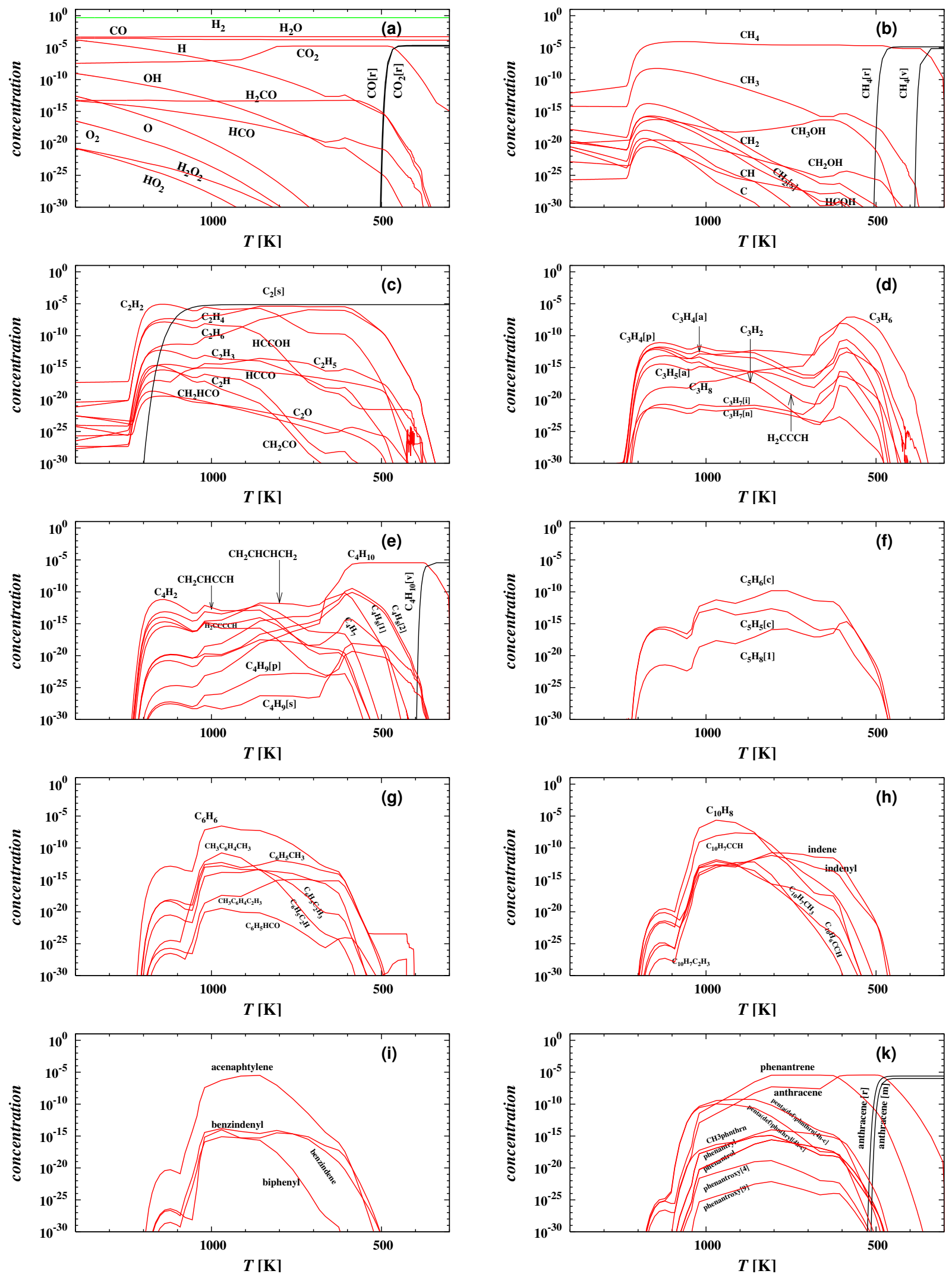

Fig. A.1. Concentration relative to $\mathrm{H}$ nuclei of the most abundant species of the $\mathrm{HCO}$ chemistry for oxidation of the carbonaceous material in the solar nebula to $\mathrm{CO}$ by water vapour. Note that the temperature increases from the right to the left. $a$ ) The water chemistry and the chemistry of $\mathrm{CO}_{2}$ and $\left.\mathrm{H}_{2} \mathrm{CO} . b\right)-f$ ) The chemistry of hydrocarbons with 1-5 C atoms. $g$ ) The chemistry of hydrocarbons with 6 and $7 \mathrm{C}$ atoms. $h$ ) The chemistry of hydrocarbons with 8-10 C atoms. $i$ ) The chemistry of hydrocarbons with 12 and $13 \mathrm{C}$ atoms. $k$ ) The chemistry of hydrocarbons with 14 and $15 \mathrm{C}$ atoms. The black lines correspond to the components of the solid carbonaceous material. 
solid carbon material. The oxidation reaction can be written with this assumption as

$$
\mathrm{C}_{2}[\mathrm{~s}]+\mathrm{OH} \longrightarrow \mathrm{HCCO} \text {. }
$$

If the carbon dust particles are spheres of radius $a$, the relation between the particle density of the species $\mathrm{C}_{2}$ [s] and the number density $n_{\text {car }}$ of carbon grains is

$$
\frac{4 \pi a^{3}}{3} \rho_{\mathrm{car}} n_{\mathrm{car}}=2 A_{\mathrm{C}} m_{\mathrm{H}} n_{\mathrm{C}_{2}[\mathrm{~s}]}^{(\mathrm{s})} \text {, }
$$

where $A_{\mathrm{C}}$ is the atomic weight of carbon and $\rho_{\text {car }}$ the mass density of solid carbon.

The rate for the starter reaction of soot oxidation is

$$
\frac{R_{\mathrm{car}}}{n_{\mathrm{H}}}=k_{\mathrm{car}} n_{\mathrm{OH}} c_{\mathrm{C}_{2}[\mathrm{~s}]}^{(\mathrm{s})} \text {, }
$$

with $c_{\mathrm{C}_{2}[\mathrm{~s}]}^{(\mathrm{s})}$ being the particle density of $\mathrm{C}$ atoms bound in soot, $n_{\mathrm{OH}}$ the particle density of $\mathrm{OH}$ radicals, and $k_{\mathrm{car}}$ the corresponding rate coefficient, which can be written as

$k_{\mathrm{car}}=\frac{3 v_{\mathrm{OH}} \alpha 2 A_{\mathrm{C}} m_{\mathrm{H}}}{a \rho_{\mathrm{car}}}$.

Here $v_{\mathrm{th}, \mathrm{OH}}$ is the rms thermal velocity of $\mathrm{OH}$,

$v_{\mathrm{th}, \mathrm{OH}}=\sqrt{\frac{k T}{2 \pi m_{\mathrm{OH}}}}$,

and $\alpha_{\text {car }}(=0.1)$ is the efficiency for the reaction of $\mathrm{OH}$ with solid carbon (see Finocchi et al. 1997, for this). Writing this as

$k_{\mathrm{car}}=A T^{b}$,

we obtain the numerical coefficients $A, b$ for the rate coefficient given in Table A.2, with an assumed radius of $a=5 \times 10^{-6}$ (see Sect. 2.1) and density $\rho_{\text {car }}=2 \mathrm{~g} \mathrm{~cm}^{-3}$.

\section{Appendix B: Gas-phase chemistry of hydrocarbons}

The oxidation of hydrocarbons is the basic process responsible for the final conversion of the pyrolysis products into $\mathrm{CO}$. Because of their technical importance, the burning processes of organic materials are well studied within in the field of flame chemistry (e.g. Warnatz et al. 2006). The main difference between terrestrial flames and processes in an accretion disc is the high excess of hydrogen in astrophysical settings. Therefore most oxygen that is not bound in $\mathrm{CO}$ is found in $\mathrm{H}_{2} \mathrm{O}$ and its dissociation products $\mathrm{OH}$ and $\mathrm{O}$, and almost no $\mathrm{O}_{2}$ is found, which is a major oxygen carrier in technical flames. $\mathrm{CO}_{2}$ is also of low abundance, in contrast to terrestrial flames. Moreover, the pressure is lower in the solar nebula than in laboratory low-pressure flames, but not by orders of magnitude, and temperatures at which oxidation occurs are expected to be lower because of the much longer characteristic timescales for temperature changes. The general conditions under which the chemistry operates are similar, however. This means that the relative importance of the various oxidation reactions is different in the solar nebula from that in the laboratory, but the same suite of chemical compounds should be present in the solar nebula as in flames, and the same reaction mechanisms should operate in the solar nebula as those that are observed for technical burning processes under low-pressure conditions.

To model the oxidation of the carbon dust and the hydrocarbons released from the carbonaceous material, we used the basic reaction mechanisms developed for modelling sooting flames (e.g. Marinov et al. 1996; Wang \& Frenklach 1997; Appel et al. 2000). In our model calculation, the H, C, and O chemistry is based on the model for combustion and soot formation of aliphatic fuels by Marinov et al. (1996). Because low pressure prevails in the accretion disc $(\lesssim 100 \mathrm{~Pa}$, cf. Fig. 2$)$, the low-pressure limit of all pressure-dependent reactions was used. Additionally, He was included because this may be important for the few three-body reactions that may occur at the low pressures in accretion disks. The chemical reaction system used for this calculation consists of 146 species formed from $\mathrm{H}, \mathrm{C}$, and $\mathrm{O}$. It contains the $\mathrm{OH}$-system for the formation and dissociation of water with 8 species and 138 species with 118 carbon atoms and the entire 1341 chemical reactions from Marinov et al. (1996), including the automatically generated reverse reactions, where necessary.

\section{Appendix C: Sample calculation}

We considered the oxidation of the mixture of carbonaceous materials assumed to have existed in the solar nebula. We added the components $\mathrm{CH}_{4}[\mathrm{v}]$ to $\mathrm{C}_{16} \mathrm{H}_{10}[\mathrm{r}]$ as defined in Appendix A.1 and the equations for their pyrolysis to the reaction network for the combustion chemistry of aliphatic fuels. We used the rate coefficients as defined by Table A.2 for their pyrolysis. The initial concentrations of the gas-phase species were chosen as follows: all $\mathrm{H}$ is in $\mathrm{H}_{2}$, one half of the $\mathrm{C}$ abundance in $\mathrm{CO}$, and the part of the $\mathrm{O}$ that is not bound in minerals and $\mathrm{CO}$ is in $\mathrm{H}_{2} \mathrm{O}$. The concentration of all other gas phase species was set to zero. The corresponding element abundances are given in Table 3 . The initial concentrations of the components released at elevated temperature by pyrolysis are defined by Eq. (1). The complete set of reaction equations was solved numerically by a fully implicit Euler method. We started with an initial temperature of $300 \mathrm{~K}$ and increased the temperature at a fixed rate of $10^{-2} \mathrm{~K} \mathrm{yr}^{-1}$ up to $1400 \mathrm{~K}$. The rate of the temperature increase was chosen such that it corresponded approximately to the temperature change during the accretion flow at about 1 AU distance in the disc model from Sect. 6.1. A pressure of $p=10^{-4}$ bar was assumed.

The resulting variation of the concentrations of the species is shown in Fig. A.1, where the concentration of species relative to $\mathrm{H}$-nuclei from the $\mathrm{HO}$-chemistry and the species related to the $\mathrm{CO}-\mathrm{CO}_{2}$-chemistry are shown in Fig. A.1a, while Figs. A.1b-k show the hydrocarbon species with 1 to 15 carbon atoms. The black lines show the different component concentrations of the solid carbonaceous material. These components disappear between $\sim 350$ and $\sim 500 \mathrm{~K}$ by pyrolysis, except for the amorphous carbon, which is oxidised at about $1200 \mathrm{~K}$ by reactions with $\mathrm{OH}$ radicals from the gas phase.

The liberation of the pyrolysis products results in a rich gasphase chemistry of numerous hydrocarbon compounds from the whole size range from 1 to 15 carbon atoms. Even compounds with a higher number of $\mathrm{C}$ atoms are predicted to be formed when the network is extended to such compounds. This is in so far remarkable as in the oxygen-rich and hydrogen-rich environment of the solar nebula, none of them would exist in abundance under chemical equilibrium conditions; $\mathrm{CO}$ would be the main carbon-bearing gas phase species. The reason why such a rich zoo of hydrocarbons exists is that all possible oxidation reactions that finally convert hydrocarbons into $\mathrm{CO}$ involve rather high activation energies and do not become operative under the low-pressure conditions prevailing in the solar nebula until the temperature exceeds about $1200 \mathrm{~K}$. At lower temperatures, the hydrocarbon chemistry cannot evolve into chemical 
equilibrium, and hydrocarbons can coexist with water vapour in a metastable state (Zolotov \& Shock 2001). Reactions between hydrocarbons and atomic $\mathrm{H}$ occur with significant rates such that all hydrocarbons with more than three carbon atoms are degraded to small hydrocarbons with 1 or 2 carbon atoms, mainly $\mathrm{CH}_{4}$ and $\mathrm{C}_{2} \mathrm{H}_{2}$, before they disappear at temperatures above $\sim 1200 \mathrm{~K}$ by oxidation to $\mathrm{CO}$. The final stage of the hydrocarbon combustion involves only compounds with one or two carbon atoms. This is a well-known phenomenon in the combustion of organic fuels. More details of the gas-phase chemistry will be discussed elsewhere.
The important point is that the amorphous carbon component does not vanish from the dust component in the solar nebula before the $\mathrm{OH}$ concentration climbs to a level at which the reaction rate (A.4) becomes sufficiently high. This requires temperatures of $T \gtrsim 1200 \mathrm{~K}$. Planetesimals formed in disc regions with lower temperature will incorporate the residual amorphous carbon at the onset of planetesimal formation.

These follow-up processes of the gasification of the carbonaceous material will be discussed in more detail elsewhere. 